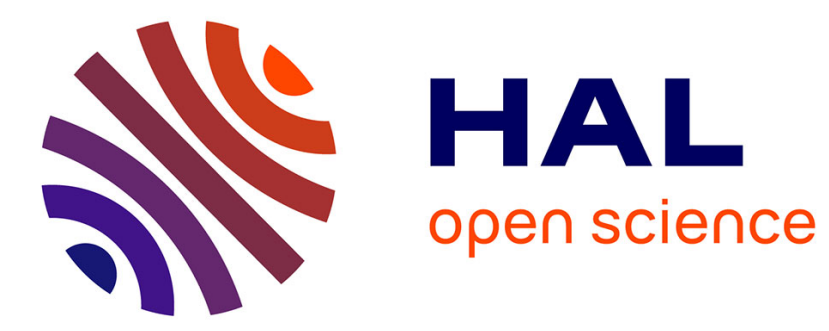

\title{
Stabilization by a relay control using non-quadratic Lyapunov functions
}

Zohra Kader, Christophe Fiter, Laurentiu Hetel, Lotfi Belkoura

\section{To cite this version:}

Zohra Kader, Christophe Fiter, Laurentiu Hetel, Lotfi Belkoura. Stabilization by a relay control using non-quadratic Lyapunov functions. Automatica, 2018, 97, pp.353 - 366. 10.1016/j.automatica.2018.08.020 . hal-01871693

\section{HAL Id: hal-01871693 \\ https://inria.hal.science/hal-01871693}

Submitted on 11 Sep 2018

HAL is a multi-disciplinary open access archive for the deposit and dissemination of scientific research documents, whether they are published or not. The documents may come from teaching and research institutions in France or abroad, or from public or private research centers.
L'archive ouverte pluridisciplinaire HAL, est destinée au dépôt et à la diffusion de documents scientifiques de niveau recherche, publiés ou non, émanant des établissements d'enseignement et de recherche français ou étrangers, des laboratoires publics ou privés. 


\title{
Stabilization by a relay control using non-quadratic Lyapunov functions ${ }^{\star}$
}

\author{
Zohra Kader ${ }^{\mathrm{a}, \mathrm{b}, *}$ Christophe Fiter ${ }^{\mathrm{a}}$ Laurentiu Hetel ${ }^{\mathrm{c}}$ Lotfi Belkoura ${ }^{\mathrm{a}, \mathrm{b}}$

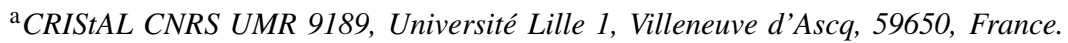 \\ ${ }^{\mathrm{b}}$ Non-A, INRIA - Lille Nord Europe, 40 avenue Halley, Villeneuve d'Ascq, 59650, France. \\ ${ }^{\mathrm{c}}$ CRIStAL CNRS UMR 9189, École Centrale de Lille, Villeneuve d'Ascq, 59650, France.
}

\begin{abstract}
In this article we consider the stabilization problem by a relay control using non-quadratic Lyapunov functions. First, a general result is proposed for the case of nonlinear systems. A full state relay feedback controller is designed in order to ensure the local asymptotic stability of the closed-loop system. Then, the result is applied to the particular case of LTI systems. A constructive method based on LMI conditions is given in order to design nonlinear switching surfaces and provide an estimation of a non-ellipsoidal domain of attraction. In addition, the approach is extended to observer-based relay feedback. Both linear and nonlinear switching surfaces dependent on the estimated state are designed while using a Luenberger observer. Finally, illustrative examples are proposed in order to show the efficiency of the proposed methods and simulations are performed for a Buck converter structure.
\end{abstract}

Key words: relay control, linear time invariant systems, nonlinear switching surfaces, switched affine systems, local asymptotic stabilization.

\section{Introduction}

Relay feedback systems present the simplest class of switched control systems [33]. They are widely used in different application fields - see for instance [23], [34], [49], [50]. They are motivated by their use in simple electrical (DC-DC converters) [14], [15], electromechanical [3], [47], and aerospace applications [18], [19]. They are also used for quantization errors modelling in digital control [33], [39], delta-sigma modulator design in signal processing [44], and controllers auto-tuning [23].

Relay systems are known for being simple, efficient and robust [16], [26], [41]. However, they may present complex phenomena which make their study very challenging : zeno solutions, limit cycles, chattering or sliding modes (see for instance [21], [28], [29], and [48]). They are related to hybrid systems and more particularly to switched affine systems [11], [13], [20], [24], [31]. Various approaches have been proposed for relay feedback control design in the litera-

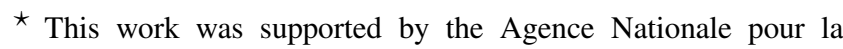
Recherche Projet ROCC-SYS under the Grant ANR-14-C27-0008.

* Corresponding author.

Email address: zohra.kader@inria.fr (Zohra Kader).
}

ture, both in the space domain and in the frequency domain see for instance [9], [26], [40], and [41]. However, the problem of relay feedback control design is still widely open. Recently, a convex embedding formalism has been used in order to design a stabilizing switching law [26]. The design procedure uses the existence of an exponentially globally stabilizing state feedback controller as a reference control to be emulated by a relay feedback controller.

The work in this paper is in the same spirit. We generalize the approach in several directions. In [26] consideration is given to the stabilization of linear systems by relay feedback controllers. Here, we propose a general framework for the design of a relay feedback control in the case of nonlinear input-affine systems. The designed switching surfaces in [26] are linear and local stabilization of the closed-loop system is ensured in an ellipsoidal domain of attraction. In this paper we propose a numerical method for the design of nonlinear switching surfaces using non-quadratic Lur'e type Lyapunov functions. Local stability of the closed-loop system is guaranteed in a larger non-ellipsoidal domain of attraction. Since the state variables in real systems are not always fully available to measurements, the approach is generalized to observer-based control. To the best of our knowledge, no observer-based relay control design method exists in the literature. We also provide a separation principle for 
LTI systems with an observer-based relay controller while, to the best of our knowledge, the separation principle exists only for systems with continuous controller [43] and thus can not be applied to relay systems. In order to illustrate the efficiency of the proposed methods a numerical example is used and simulations are performed for a Buck converter structure.

The result can be related to the simplex method [7]. We use numerical tools which are inspired from convex optimization approaches employed for systems with saturated actuators [8], [10], [45] and LMIs [1], [2], [35].

The paper is structured as follows: Section 2 gives some preliminaries and exposes the problem under study. In Section 3 we consider the case of full state relay feedback control design. The section is organized as follows : in the first part a general result is given for the local asymptotic stabilization of nonlinear affine systems with relay feedback. In the second part, we propose an LMI based approach allowing the design of nonlinear state-dependent switching laws that ensure the stability of LTI systems and provide a nonellipsoidal estimation of the domain of attraction. In addition, a numerical example that illustrates the efficiency of the proposed method is also provided in the same section. Design methods of observer-based relay feedback controller are proposed in Section 4. We consider both linear and nonlinear switching laws dependent on the estimated state using a Luenberger observer. Computer simulations are performed for a Buck converter structure in order to asses the effectiveness of the proposed approaches. The paper is ended with a brief conclusion.

\subsection{Notations}

In this paper we use the notation $\mathbb{R}_{+}$to refer to the interval $[0, \infty)$. The transpose of a matrix $M$ is denoted by $M^{T}$ and if the matrix is symmetric the symmetric elements are denoted by $*$. The notation $M \succeq 0$ (resp. $M \preceq 0$ ) means that the matrix $M$ is positive (resp. negative) semi-definite, and the notation $M \succ 0$ (resp. $M \prec 0$ ) means that it is positive definite (resp. negative definite). The identity matrix is denoted by $I$ and both notations $\operatorname{eig}_{\text {min }}(M)$ and $\operatorname{eig}_{\text {max }}(M)$ are used to refer to the minimum and maximum eigenvalue respectively of a matrix $M$. The notations $M_{(i)}$ and $M_{(i, j)}$ refer respectively to the $i$-th row of a matrix (or vector) $M$, and the element of the $i$-th row and the $j$-th column of a matrix $M$.

For a positive integer $N$, we denote by $\mathscr{I}_{N}$ the set $\{1, \ldots, N\}$. By $\Delta_{N}$ we denote the unit simplex

$$
\Delta_{N}=\left\{\beta=\left[\begin{array}{c}
\beta_{(1)} \\
\vdots \\
\beta_{(N)}
\end{array}\right] \in \mathbb{R}^{N}: \sum_{i=1}^{N} \beta_{(i)}=1, \beta_{(i)} \geq 0, i \in \mathscr{I}_{(N)}\right\}
$$

A vector $v \in \mathbb{R}^{m}$ is said to be strictly positive if for all $i \in \mathscr{I}_{m}$ $v_{(i)}>0$.
For a positive definite matrix $P \in \mathbb{R}^{n \times n}$ and a positive scalar $\gamma$, we denote by $\mathscr{E}(P, \gamma)$ the ellipsoid

$$
\mathscr{E}(P, \gamma)=\left\{x \in \mathbb{R}^{n}: x^{T} P x \leq \gamma\right\},
$$

and for all positive scalar $r$, we denote by $\mathscr{B}(0, r)$ the ball of radius $\sqrt{r}$

$$
\mathscr{B}(0, r)=\mathscr{E}(I, r)=\left\{x \in \mathbb{R}^{n}: x^{T} x \leq r\right\} .
$$

For a given set $\mathscr{S}$, the notation $\operatorname{Conv}\{\mathscr{S}\}$ indicates the convex hull of the set, $\operatorname{int}\{\mathscr{S}\}$ its interior and $\overline{\mathscr{S}}$ its closure. The closed convex hull of the set $\mathscr{S}$ will be noted by $\overline{\operatorname{Conv}}\{\mathscr{S}\}$. Finally, we denote by Vert $\{\mathscr{S}\}$ the set of vertices of $\mathscr{S}$. Let $\mathscr{S} \subset \mathbb{R}^{m}$ be a finite set of vectors. The minimum argument of a given function $f: \mathscr{S} \longrightarrow \mathbb{R}$ is noted by

$$
\underset{x \in \mathscr{S}}{\arg \min } f(x)=\{y \in \mathscr{S}: f(y) \leq f(z), \forall z \in \mathscr{S}\} .
$$

\section{Preliminaries}

\subsection{System description}

Consider the linear system

$$
\begin{aligned}
& \dot{x}=A x+B u, \\
& x(0)=x_{0} \in \mathbb{R}^{n},
\end{aligned}
$$

with $x \in \mathbb{R}^{n}$, and an input $u$ which takes values in the set $\mathscr{V}=\left\{v_{1}, \ldots, v_{N}\right\} \subset \mathbb{R}^{m} . A \in \mathbb{R}^{n \times n}$ and $B \in \mathbb{R}^{n \times m}$ are the matrices describing the system. In the sequel we assume that:

A-1 The pair $(A, B)$ is stabilizable. This means that there exists a matrix $K$ such that the closed-loop matrix $A_{c l}=$ $A+B K$ is Hurwitz.

A-2 The set $\operatorname{int}\{\operatorname{Conv}\{\mathscr{V}\}\}$ is nonempty and the null vector is contained inside $(0 \in \operatorname{int}\{\operatorname{Conv}\{\mathscr{V}\}\})$.

Note that for any finite set $\mathscr{V}$ there exists a finite number $n_{l}$ of vectors $l_{i} \in \mathbb{R}^{1 \times m}, i \in \mathscr{I}_{n_{l}}$ such that

$$
\operatorname{Conv}\{\mathscr{V}\}=\left\{u \in \mathbb{R}^{m}: l_{i} u \leq 1, \forall i \in \mathscr{I}_{n_{l}}\right\}
$$

Note also that typical control sets $\mathscr{V}$ are often of the form

$$
\mathscr{V}=\operatorname{Vert}\{\mathscr{P}(c)\}
$$

where the hyperrectangle $\mathscr{P}(c)$, with $c$ a strictly positive vector, is given by

$$
\mathscr{P}(c)=\left\{u=\left[\begin{array}{c}
u_{(1)} \\
\vdots \\
u_{(m)}
\end{array}\right] \in \mathbb{R}^{m}:\left|u_{(i)}\right| \leq c_{(i)}, \forall i \in \mathscr{I}_{m}\right\}
$$


However, we want to keep the problem formulation as general as possible. Note that since A.2 holds, even for more general sets $\mathscr{V}$ there exists a vector $c \in \mathbb{R}^{m}$ such that the hyperrectangle satisfies $\mathscr{P}(c) \subseteq \operatorname{Conv}\{\mathscr{V}\}$. In the sequel, we will consider such a vector $c$ and use the notation (7) to prove the results.

This paper deals with the stabilization of system (4). We consider controllers described by

$$
u(x) \in \underset{v \in \mathscr{V}}{\arg \min } \Gamma(x, v),
$$

where the mapping $\Gamma: \mathbb{R}^{n} \times \mathscr{V} \longrightarrow \mathbb{R}$ characterizes the switching surfaces.

This formulation encompasses the classical sign function in the classical relay feedback controller. Indeed, if $\mathscr{V}=$ $\{-v, v\}$ with $v>0$ and $\Gamma(x, v)=x^{T} \Psi v$ for some $\Psi \in \mathbb{R}^{n \times m}$, we get

$$
u(x)=\left\{\begin{array}{l}
v, \quad \text { if } \quad \Psi^{T} x \leq 0 \\
-v, \quad \text { if } \quad \Psi^{T} x>0
\end{array}\right.
$$

The interconnection (4), (8) can be rewritten as follows

$$
\dot{x}=A x+B u(x)=\mathscr{X}(x) .
$$

Note that this is a differential equation with a discontinuous right hand side $\mathscr{X}(x)$ [12], [17] and thus we need an appropriate formalism and specific tools to define the system's solutions and analyse their behaviour, in particular when sliding dynamics occur.

Therefore, in order to take into account possible sliding motions, to the discontinuous closed-loop system (10) we associate the differential inclusion

$$
\dot{x} \in \mathscr{F}[\mathscr{X}](x),
$$

with $\mathscr{F}[\mathscr{X}](x)$ the set-valued map which can be computed from the differential equation with a discontinuous right hand side using the construction given by Filippov [17] (see also [5], [12], and [42])

$$
\mathscr{F}[\mathscr{X}](x)=\bigcap_{\delta>0} \bigcap_{\mu(\mathscr{S})=0} \overline{\operatorname{Conv}}\{\mathscr{X}(\tilde{\mathscr{B}}(x, \delta) \backslash \mathscr{S})\}, \forall x \in \mathbb{R}^{n},
$$

where $\tilde{\mathscr{B}}(x, \delta)$ is the open ball centered on $x$ with radius $\sqrt{\delta}$, and $\mathscr{S}$ is a set of measure (in the sense of Lebesgue) $\mu(\mathscr{S})=0$.

System (10) is a subclass of the general class of time-varying systems given by

$$
\dot{x}=\overline{\mathscr{X}}(t, x), x\left(t_{0}\right)=x_{0},
$$

where $\overline{\mathscr{X}}(t, x)$ is locally bounded and Lebesgue measurable with respect to $(t, x) \in[0,+\infty) \times \mathbb{R}^{n}$.
To this system we associate the following time-varying differential inclusion

$$
\dot{x} \in \mathscr{F}[\overline{\mathscr{X}}](t, x), x\left(t_{0}\right)=x_{0},
$$

with

$$
\begin{aligned}
& \mathscr{F}[\overline{\mathscr{X}}](t, x)= \\
& \bigcap_{\delta>0} \bigcap_{\mu(\mathscr{S})=0} \overline{\operatorname{Conv}}\{\overline{\mathscr{X}}(t, \tilde{\mathscr{B}}(x, \delta) \backslash \mathscr{S})\}, \forall x \in \mathbb{R}^{n}, t \in \mathbb{R}_{+} .
\end{aligned}
$$

The notion of solution of a differential inclusion was defined in [17], and is recalled hereafter.

Definition 1 (Filippov solution) Consider the differential inclusion (14). A Filippov solution of the discontinuous system (13) over the interval $\left[t_{a}, t_{b}\right] \subset[0, \infty)$ is an absolutely continuous mapping $\varsigma(t):\left[t_{a}, t_{b}\right] \longrightarrow \mathbb{R}^{n}$ satisfying:

$$
\begin{aligned}
& \qquad \dot{\zeta}(t) \in \mathscr{F}[\overline{\mathscr{X}}](t, \varsigma(t)), \text { for almost all } t \in\left[t_{a}, t_{b}\right], \\
& \text { with } \mathscr{F}[\overline{\mathscr{X}}](t, x) \text { given by }(15) \text {. }
\end{aligned}
$$

A differential inclusion has at least one solution if the set valued map $\mathscr{F}[\bar{X}](t, x)$ is nonempty, locally bounded, closed, convex, and $\mathscr{F}$ is upper semicontinuous on $x, t$ [4], [5], [12], [17].

Definition 2 (Equilibrium point) $x_{e q}$ is said to be an equilibrium point of the differential inclusion (14) (resp. of differential inclusion (11)) if $0 \in \mathscr{F}[\overline{\mathscr{X}}]\left(t, x_{\text {eq }}\right)$ for all $t \geq t_{0} \geq 0$ (resp. if $0 \in \mathscr{F}[\mathscr{X}]\left(x_{\text {eq }}\right)$ ).

Hereafter the notions of stability which will be used are introduced and we mathematically formalize the problem under study.

Definition 3 (Stability concepts) The equilibrium point $x=0$ of the differential inclusion (14) is said to be :

(1) uniformly stable, if for any $\varepsilon>0$, there exists $\delta>0$, independent of $t_{0}$, such that for all Filippov solutions $x(t)$ of (14), $\left\|x\left(t_{0}\right)\right\|<\delta \Longrightarrow\|x(t)\|<\varepsilon, \forall t \geq t_{0} \geq 0$,

(2) locally uniformly asymptotically stable, if it is uniformly stable and there exists a set $\hat{\mathscr{D}} \subset \mathbb{R}^{n}, 0 \in \operatorname{int}\{\hat{\mathscr{D}}\}$, such that for all Filippov solutions $x(t)$ of (14) with $x\left(t_{0}\right) \in \hat{\mathscr{D}}, x(t) \longrightarrow 0$ when $t \longrightarrow \infty$,

(3) locally uniformly exponentially stable with a decay rate $\alpha$ (or locally uniformly $\alpha$-stable), if there exist a set $\hat{\mathscr{D}} \subset \mathbb{R}^{n}, 0 \in \operatorname{int}\{\hat{\mathscr{D}}\}$, and strictly positive scalars $\kappa$ independent of $t_{0}$, and $\alpha$ such that for all Filippov solutions $x(t)$ of (14) with $x\left(t_{0}\right) \in \hat{\mathscr{D}}$,

$$
\|x(t)\| \leq \kappa e^{-\alpha\left(t-t_{0}\right)}\left\|x\left(t_{0}\right)\right\|, \forall t \geq t_{0} \geq 0 .
$$

A set $\hat{\mathscr{D}}$ satisfying one of these properties is usually called an estimation of the domain of attraction. 
For the case of time invariant systems (11) (or equivalently $(10),(8))$ the stability properties do not depend on the time variable $t$. Therefore, the uniformity property can be omitted for this class of systems.

We recall (see [17], Chapter 3, page 153, Theorem 1) that sufficient conditions for the local uniform asymptotic stability of Filippov solutions in the case of systems modelled by a differential equation with a discontinuous right hand side $\dot{x}=\bar{X}(t, x)$, are given by the existence of a strict Lyapunov function $V, V(t, 0)=0, V(t, x) \geq 0, \forall x \neq 0, t \geq t_{0} \geq 0$ and continuous positive definite functions $W_{1}, W_{2}$, and $W_{3}$ (i.e. $W_{i}(0)=0$ and $W_{i}(x)>0$ for all $x \neq 0$ and $\left.i \in\{1,2,3\}\right)$, such that

$$
W_{1}(x) \leq V(t, x) \leq W_{2}(x),
$$

and

$\sup _{\varsigma \in \mathscr{F}[\mathscr{X}](t, x)}\left\{\frac{\partial V}{\partial t}+\frac{\partial V}{\partial x} \varsigma\right\} \leq-W_{3}(x), \forall x \in \mathscr{D} \backslash\{0\}, \forall t \geq 0$,

for some domain $\mathscr{D}$ such that $0 \in \mathscr{D}$. For the case of local uniform exponential stability, sufficient conditions can be obtained from (18) and (19), by considering a particular form of $W_{3}$ such that $W_{3}(x) \geq 2 \alpha V(t, x), \forall t \geq 0$, where $\alpha$ is a positive scalar.

In [17], Chapter 3, page 153, Theorem 1, it is also required that the mapping $\mathscr{F}[\mathscr{\mathscr { X }}](t, x)$ satisfies the "basic conditions" cited in [17], Chapter 2, page 76, point 2, in order to guarantee the system's local uniform asymptotic (or exponential) stability. With the function $\overline{\mathscr{X}}(t, x)$ defining the system (13) locally bounded and Lebesgue measurable, as we have assumed, it has been shown in [17], Chapter 2, page 85, point 6 , that the set-valued map $\mathscr{F}[\mathscr{X}](t, x)$ defined in (14)-(15) satisfies those "basic conditions".

Using these notations and definitions, we are now able to state the problem under study in this article.

\subsection{Recent results and problem statement}

In this paper we are interested in the study of the following problem:

Problem 1. Given system (4) under Assumptions A-1, A-2 and the set $\mathscr{V}$, design a relay feedback controller (8) such that the origin of the closed-loop system is locally asymptotically stable.

In [25] a constructive method for a relay feedback controller design is given. Assuming A-2 and

A-1' There exist a positive definite matrix $Q$ and positive scalars $\chi$ and $\alpha$ such that

$$
A Q+Q A^{T}-\chi B B^{T} \preceq-2 \alpha Q
$$

it is proved that system (4) with a switching law (8) is locally exponentially stable with a decay rate $\alpha$. Note that A-1' is equivalent to A-1. In [25], a linear switching function is considered: $\Gamma(x, v)=-\frac{2}{\chi} x^{T} H^{T} v$ with $H=-\frac{\chi}{2} B^{T} Q^{-1}$. An ellipsoidal estimation of the domain of attraction is equally given using a quadratic Lyapunov function $V(x)=x^{T} P x$ with $P=Q^{-1}: \mathscr{E}(P, \gamma)$ where $\gamma$ is computed such that $\mathscr{E}(P, \gamma)$ does not cross the convex hull

$$
\mathscr{C}_{v}(H)=\left\{x \in \mathbb{R}^{n}: l_{i} H x \leq 1, \forall i \in \mathscr{I}_{n_{l}}\right\},
$$

where $l_{i}, i \in \mathscr{I}_{n_{l}}$ are vectors defined in (5), which leads to $\gamma \leq$ $\min _{i \in \mathscr{I}_{n_{l}}}\left(l_{i} H Q H^{T} l_{i}^{T}\right)^{-1}$. Nevertheless, considering a quadratic Lyapunov function, linear switching surfaces and an ellipsoidal estimation of the domain of attraction introduces some conservatism in the proposed method [8].

Here we would like to provide a more general design procedure using non-quadratic Lyapunov functions to compute nonlinear switching surfaces and non-ellipsoidal estimations of the domain of attraction.

With the above definitions, in the next section, we start by providing sufficient conditions for nonlinear input-affine systems stabilization with a relay feedback controller. A constructive method based on LMI criteria is also provided for the class of LTI systems. It allows the design of nonlinear stabilizing state-dependent switching laws and provides a larger estimation of the domain of attraction.

\section{Full state relay feedback control design}

\subsection{A general theoretical result}

Before considering the case of linear systems, here we provide a general result for nonlinear systems stabilization with a relay controller.

The method uses the existence of a locally stabilizing continuous control to design the relay controller.

Consider the following nonlinear input-affine system

$$
\dot{x}=f(t, x)+g(t, x) u(t, x)=\overline{\mathscr{X}}(t, x),
$$

where $f: \mathbb{R}_{+} \times \mathscr{D} \longrightarrow \mathbb{R}^{n}, g: \mathbb{R}_{+} \times \mathscr{D} \longrightarrow \mathbb{R}^{n \times m}$ are Lipschitz functions in $x$ on $\mathbb{R}_{+} \times \mathscr{D}$ and piecewise continuous in $t$, and where $\mathscr{D} \subset \mathbb{R}^{n}$ is a domain such that $0 \in \operatorname{int}\{\mathscr{D}\}$. The input $u$ takes values in the set $\mathscr{V}=\left\{v_{1}, \ldots, v_{N}\right\} \subset \mathbb{R}^{m}$.

Using the above definitions we are now able to provide sufficient conditions for the stabilization of the nonlinear timevarying system (22) by relay controller.

Theorem 1 Consider the nonlinear system (22) and Assumption A-2. Assume that there exists a continuous controller $k(t, x)$ such that $k(t, x) \in \operatorname{Conv}\{\mathscr{V}\}$ and $g(t, x) k(t, x)$ 
is Lipschitz in $x$ on $\mathbb{R}_{+} \times \mathscr{D}$ and piecewise continuous in $t$, for all $x \in \mathscr{D}$ and for all $t \geq 0$. Assume that there exists a continuously differentiable function $V: \mathbb{R}_{+} \times \mathscr{D} \longrightarrow \mathbb{R}$ such that

$$
\begin{aligned}
W_{1}(x) \leq V(t, x) & \leq W_{2}(x) \\
\frac{\partial V}{\partial t}+\frac{\partial V}{\partial x}\{f(t, x)+g(t, x) k(t, x)\} & \leq-W_{3}(x), \forall t \geq 0, \forall x \in \mathscr{D},
\end{aligned}
$$

where $W_{1}, W_{2}$ and $W_{3}$ are continuous positive definite functions on $\mathscr{D}$. Then, the origin of system (22) with the discontinuous controller

$$
u(t, x)=k^{d}(t, x) \in \underset{v \in \mathscr{V}}{\arg \min } \frac{\partial V}{\partial x} g(t, x) v, \forall t \geq 0, \forall x \in \mathscr{D},
$$

is locally uniformly asymptotically stable. Moreover, the set $\mathscr{L}_{V}(\bar{\eta})=\left\{x \in \mathbb{R}^{n}: V(t, x) \leq \bar{\eta}, \forall t \geq 0\right\} \subseteq \mathscr{D}$ with $\bar{\eta}=$ $\max \left\{\eta>0: \mathscr{L}_{V}(\eta) \subseteq \mathscr{D}\right\}$ is an estimation of the domain of attraction.

Proof. We would like to prove that the origin of the closedloop system

$$
\dot{x}=f(t, x)+g(t, x) k^{d}(t, x)
$$

is locally uniformly asymptotically stable when solutions are understood in the sense of Filippov. Since $\frac{\partial V}{\partial x}$ and $g(t, x)$ are continuous, then $\operatorname{Conv}\left\{\underset{v \in \mathscr{V}}{\arg \min } \frac{\partial V}{\partial x} g(t, x) v\right\}$ is upper semicontinuous. Therefore, we consider the following differential inclusion [17], [22], [38]

$$
\dot{x} \in \mathscr{F}(t, x),
$$

with

$$
\mathscr{F}(t, x)=\operatorname{Conv}\left\{f(t, x)+g(t, x) u^{d}: u^{d} \in \underset{v \in \mathscr{V}}{\arg \min } \frac{\partial V}{\partial x} g(t, x) v\right\} .
$$

The origin of the differential inclusion (27) is locally uniformly asymptotically stable if for a given Lyapunov function $V(t, x)$ we have

$$
\sup _{\varsigma \in \mathscr{F}(t, x)}\left\{\frac{\partial V}{\partial t}+\frac{\partial V}{\partial x} \varsigma\right\} \leq-W_{3}(x), \forall t \geq 0, \forall x \in \mathscr{D} .
$$

Thanks to the fact that $k(t, x) \in \operatorname{Conv}\{\mathscr{V}\}$ for all $t \geq 0$ and for all $x \in \mathscr{D}$, there exist $N$ scalars $\rho_{i}(t, x) \geq 0, i \in \mathscr{I}_{N}$ with $\sum_{i=1}^{N} \rho_{i}(t, x)=1$ such that

$$
k(t, x)=\sum_{i=1}^{N} \rho_{i}(t, x) v_{i}
$$

Considering (30) and replacing $k(t, x)$ by $\sum_{i=1}^{N} \rho_{i}(t, x) v_{i}$, in (24) we obtain

$$
\frac{\partial V}{\partial t}+\frac{\partial V}{\partial x}\left(f(t, x)+g(t, x) \sum_{i=1}^{N} \rho_{i}(t, x) v_{i}\right)+W_{3}(x) \leq 0 .
$$

Then, using the fact that $\sum_{i=1}^{N} \rho_{i}(t, x)=1$, we get

$$
\sum_{i=1}^{N} \rho_{i}(t, x)\left(\frac{\partial V}{\partial t}+\frac{\partial V}{\partial x}\left(f(t, x)+g(t, x) v_{i}\right)+W_{3}(x)\right) \leq 0,
$$

for all $t \geq 0$ and for all $x \in \mathscr{D}$.

Let us define the function

$$
F\left(t, x, v_{i}\right)=\frac{\partial V}{\partial t}+\frac{\partial V}{\partial x}\left(f(t, x)+g(t, x) v_{i}\right)+W_{3}(x) .
$$

Since $\rho_{i}(t, x) \geq 0, \forall i \in \mathscr{I}_{N}$ and from inequality (32), it can be inferred that the inequality

$$
F\left(t, x, v_{i}\right) \leq 0, \forall x \in \mathscr{D}, \forall t \geq 0
$$

holds at least for one index $i(t, x) \in \mathscr{I}_{N}$. We can then define the switching controller as follows

$$
k^{d}(t, x) \in \underset{v \in \mathscr{V}}{\arg \min } F(t, x, v)=\underset{v \in \mathscr{V}}{\arg \min } \frac{\partial V}{\partial x} g(t, x) v .
$$

Let us define the set of minimizers corresponding to the controller (35) as follows

$$
\mathscr{I}(t, x)=\left\{i \in \mathscr{I}_{N}: \frac{\partial V}{\partial x} g(t, x)\left(v_{j}-v_{i}\right) \geq 0, \forall j \in \mathscr{I}_{N}\right\} .
$$

Consider the set valued map given by

$$
\tilde{\mathscr{F}}(t, x)=\operatorname{Conv}\left\{f(t, x)+g(t, x) v_{i}: i \in \mathscr{I}(t, x)\right\} .
$$

Since

$$
\underset{v \in \mathscr{V}}{\arg \min }\left\{\frac{\partial V}{\partial x} g(t, x) v\right\} \subseteq\left\{v_{i}: i \in \mathscr{I}(t, x)\right\}
$$

is satisfied, according to the definition of $\mathscr{I}(t, x)$ in (36), one can show that

$$
\mathscr{F}(t, x) \subseteq \tilde{\mathscr{F}}(t, x)
$$

with $\mathscr{F}(t, x)$ defined in $(28)$ and $\tilde{\mathscr{F}}(t, x)$ defined in (37).

Considering the relation (39), in order to prove (29), it is sufficient to show that

$$
\sup _{\varsigma \in \tilde{\mathscr{F}}(t, x)}\left\{\frac{\partial V}{\partial t}+\frac{\partial V}{\partial x} \varsigma\right\} \leq-W_{3}(x), \forall t \geq 0, \forall x \in \mathscr{D} .
$$

Let us define the following set of vectors

$$
\Delta(t, x)=\left\{\beta \in \Delta_{N}: \beta_{(i)}=0, \forall i \in \mathscr{I}_{N} \backslash \mathscr{I}(t, x)\right\} .
$$


We have then

$$
\begin{aligned}
\tilde{\mathscr{F}}(t, x) & =\operatorname{Conv}\left\{f(t, x)+g(t, x) v_{i}: i \in \mathscr{I}(t, x)\right\} \\
& =\left\{f(t, x)+g(t, x) \sum_{i=1}^{N} \beta_{(i)} v_{i}: \beta \in \Delta(t, x)\right\} .
\end{aligned}
$$

Consequently, since $\Delta(t, x)$ is compact, we obtain

$$
\begin{aligned}
& \sup _{\varsigma \in \mathscr{F}(t, x)}\left\{\frac{\partial V}{\partial t}+\frac{\partial V}{\partial x} \varsigma\right\} \leq \sup _{\varsigma \in \tilde{\mathscr{F}}(t, x)}\left\{\frac{\partial V}{\partial t}+\frac{\partial V}{\partial x} \varsigma\right\} \\
= & \sup _{\beta \in \Delta(t, x)}\left\{\frac{\partial V}{\partial t}+\frac{\partial V}{\partial x}\left(f(t, x)+g(t, x) \sum_{i=1}^{N} \beta_{(i)} v_{i}\right)\right\} \\
= & \max _{\beta \in \Delta(t, x)}\left\{\frac{\partial V}{\partial t}+\frac{\partial V}{\partial x}\left(f(t, x)+g(t, x) \sum_{i=1}^{N} \beta_{(i)} v_{i}\right)\right\} .
\end{aligned}
$$

Therefore, to prove (29) it is sufficient to show that

$$
\max _{\beta \in \Delta(t, x)}\left\{\frac{\partial V}{\partial t}+\frac{\partial V}{\partial x}\left(f(t, x)+g(t, x) \sum_{i=1}^{N} \beta_{(i)} v_{i}\right)\right\} \leq-W_{3}(x),
$$

for all $t \geq 0$ and for all $x \in \mathscr{D}$.

Let $\beta \in \Delta(t, x)$. Since $\beta_{(i)}=0$ for all $i \in \mathscr{I}_{N} \backslash \mathscr{I}(t, x)$, from (33), (34) (which is verified at least for one $i \in \mathscr{I}_{N}$, and then for all $i \in \mathscr{I}(t, x)$ ), and (36), we can deduce that

$$
\begin{aligned}
& \frac{\partial V}{\partial t}+\frac{\partial V}{\partial x}\left(f(t, x)+g(t, x) \sum_{i=1}^{N} \beta_{(i)} v_{i}\right) \\
& =\sum_{i=1}^{N} \beta_{(i)}\left\{\frac{\partial V}{\partial t}+\frac{\partial V}{\partial x}\left(f(t, x)+g(t, x) v_{i}\right)\right\} \\
& \leq-W_{3}(x) .
\end{aligned}
$$

Therefore, (44) (and thus (29)) is satisfied, and the origin of system (22) with the controller (25) is locally uniformly asymptotically stable.

In addition, the level set $\mathscr{L}_{V}(\eta)=\left\{x \in \mathbb{R}^{n}: V(t, x) \leq \eta, \forall t \in\right.$ $\left.\mathbb{R}_{+}\right\}$of the Lyapunov function $V$ can be considered as an inner estimation of the domain of attraction if $\eta$ is such that $\mathscr{L}_{V}(\eta) \subseteq \mathscr{D}$.

The control principle given in Theorem 1 can be used to provide constructive methods of relay feedback controllers for linear systems. Using a non-quadratic Lyapunov function, a tractable LMI approach providing an estimation of the domain of attraction and stabilizing nonlinear switching surfaces is given in the following.

\subsection{Relay feedback control design for LTI systems using nonlinear switching surfaces}

As follows we particularise the result of Theorem 1 to the case of linear systems. Our objective is to enlarge the do- main of attraction with respect to the result provided in [25] by using non-quadratic Lyapunov functions. We provide numerical tools for nonlinear switching surfaces design using Lur'e Lyapunov functions of the form

$$
V(x)=x^{T} P x-2 \sum_{j=1}^{m} \int_{0}^{H_{(j)} x} \phi_{(j)}(s) \Omega_{(j, j)} d s,
$$

with $P \in \mathbb{R}^{n \times n}$ a symmetric positive definite function, $\Omega$ a diagonal positive definite matrix, and $\phi: \mathbb{R}^{m} \longrightarrow \mathbb{R}^{m}$ a nonlinear function defined for all $y \in \mathbb{R}^{m}$ as $\phi(y)=$

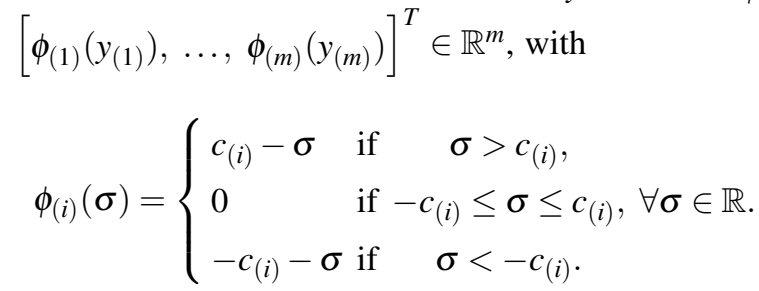

This Lyapunov class of functions has been used for various nonlinearity types (see for instance [10], [32], [45] and references therein). They have the following properties.

Lemma 1 [46] Consider $w_{1} \in \mathbb{R}^{m}$ and $w_{2} \in \mathbb{R}^{m}$. If ( $w_{1}-$ $\left.w_{2}\right) \in \mathscr{P}(c)$, with $\mathscr{P}(c)$ defined in (7), then

$$
\phi\left(w_{1}\right)^{T} M\left(\phi\left(w_{1}\right)+w_{2}\right) \leq 0
$$

for any diagonal positive definite matrix $M \in \mathbb{R}^{m \times m}$.

Lemma 2 [27] The Lur'e function (46) satisfies the inequality

$$
x^{T} P x \leq V(x) \leq x^{T}\left(P+H^{T} \Omega H\right) x, \forall x \in \mathbb{R}^{n} .
$$

Lemma 3 For any $y \in \mathbb{R}^{m}, y+\phi(y) \in \mathscr{P}(c)$, with $\mathscr{P}(c)$ defined in (7).

\section{Proof. See the Appendix.}

The Lur'e type Lyapunov function (46) is a particular class of the general Lyapunov function provided in Theorem 1. In this section we are interested in providing a constructive method based on LMIs allowing the design of the control law, along with an estimation of the domain of attraction for LTI systems.

Considering the properties provided above we are now able to develop the following result.

Theorem 2 Consider system (4) and assume that A-1' (or equivalently A-1) and A-2 hold. If there exists a symmetric positive definite matrix $P \in \mathbb{R}^{n \times n}$, two diagonal positive definite matrices $\Omega \in \mathbb{R}^{m \times m}$ and $M \in \mathbb{R}^{m \times m}$, a matrix $\Upsilon \in \mathbb{R}^{m \times n}$, and a strictly positive vector $\tau \in \mathbb{R}^{m}$ such that

$$
\left[\begin{array}{cc}
A_{c l}^{T} P+P A_{c l} & P B-\Upsilon^{T}-A_{c l}^{T} H^{T} \Omega \\
* & -2 M-\Omega H B-(\Omega H B)^{T}
\end{array}\right] \prec 0
$$


and

$$
\left[\begin{array}{cc}
P & M_{(i, i)} H_{(i)}^{T}-\Upsilon_{(i)}^{T} \\
M_{(i, i)} H_{(i)}-\Upsilon_{(i)} & \tau_{(i)} c_{(i)}^{2}
\end{array}\right] \succeq 0, \forall i \in \mathscr{I}_{m},
$$

where $H=-\frac{\chi}{2} B^{T} Q^{-1}$ and $A_{c l}=A+B H$, then the origin of system (4) with the switching law

$$
u \in \underset{v \in \mathscr{V}}{\arg \min }\left(x^{T} P-\phi(H x)^{T} \Omega H\right) B v
$$

is locally asymptotically stable.

An estimation of the domain of attraction is given by

$$
\mathscr{L}_{V}\left(r^{-1}\right)=\left\{x \in \mathbb{R}^{n}: V(x) \leq r^{-1}\right\}
$$

with $V$ a Lur'e candidate Lyapunov function given by

$$
V(x)=x^{T} P x-2 \sum_{j=1}^{m} \int_{0}^{H_{(j)} x} \phi_{(j)}(\sigma) \Omega_{(j, j)} d \sigma,
$$

and $r \geq \max _{i \in \mathscr{I}_{m}}\left\{\frac{\tau_{(i)}}{M_{(i, i)}^{2}}\right\}>0$.

Proof. The idea of the proof is to show that if A-1' and A-2 hold and the LMIs (50) and (51) are feasible then the decay of the function $V$ in the domain $\mathscr{L}_{V}\left(r^{-1}\right)$ is ensured by switching among the elements of the set $\mathscr{V}$. This will be shown in three steps. In the first step, we associate a differential inclusion to system (4), (8) and provide some sufficient conditions for local asymptotic stability of the origin. In the second step, we show that the feasibility of LMI (50) ensures the decay of the Lyapunov function in a domain $\tilde{D}$ around the origin. Finally, we will show that if the LMI (51) is feasible then the Lyapunov function decreases in the positive invariant domain $\mathscr{L}_{V}\left(r^{-1}\right) \subseteq \tilde{\mathscr{D}}$ which constitutes an inner estimation of the domain of attraction.

From Lemma 2, one can see that the Lur'e candidate Lyapunov function $V$ is positive definite.

In order to prove the local asymptotic stability of system (4), (52) in the domain $\mathscr{L}_{V}\left(r^{-1}\right)$, it is sufficient to demonstrate that

$$
\sup _{\varsigma \in \mathscr{F}[\mathscr{X}](x)} \frac{\partial V}{\partial x} \varsigma<0, \forall x \in \mathscr{L}_{V}\left(r^{-1}\right) \backslash\{0\}
$$

where $\mathscr{F}[\mathscr{X}](x)$ is defined in (12) and $\mathscr{L}_{V}\left(r^{-1}\right)$ in (53). We follow here the same steps of the proof of Theorem 1.

Let us define for all $x \in \mathbb{R}^{n}$ the set of indexes $\mathscr{I}(x)$ corresponding to the set of minimizers in which the controller (52) takes values:

$$
\mathscr{I}(x)=\left\{i \in \mathscr{I}_{N}:\left(x^{T} P-\phi(H x)^{T} \Omega H\right) B\left(v_{j}-v_{i}\right) \geq 0, \forall j \in \mathscr{I}_{N}\right\} .
$$

We associate to this set of indexes the set $\Delta(x)$ of vectors defined for all $x \in \mathbb{R}^{n}$ as

$$
\Delta(x)=\left\{\beta \in \Delta_{N}: \beta_{(i)}=0, \forall i \in \mathscr{I}_{N} \backslash \mathscr{I}(x)\right\} .
$$

Using (56) and (57) the set valued map $\mathscr{F}[\mathscr{X}](x)$ in (12) satisfies

$$
\mathscr{F}[\mathscr{X}](x) \subseteq \tilde{\mathscr{F}}[\mathscr{X}](x),
$$

with

$$
\begin{aligned}
\tilde{\mathscr{F}}[\mathscr{X}](x) & =\operatorname{Conv}_{i \in \mathscr{I}(x)}\left\{A x+B v_{i}\right\} \\
& =\{A x+B v(\beta): \beta \in \Delta(x)\},
\end{aligned}
$$

where $v(\beta)=\sum_{i=1}^{N} \beta_{(i)} v_{i}$.

From (58) and (59), and using the fact that $\Delta(x)$ is compact, we have

$$
\begin{aligned}
\sup _{\varsigma \in \mathscr{F}[\mathscr{X}](x)} \frac{\partial V}{\partial x} \varsigma & \leq \sup _{\varsigma \in \tilde{\mathscr{F}}[\mathscr{X}](x)} \frac{\partial V}{\partial x} \varsigma \\
& =\sup _{\beta \in \Delta(x)}\left\{\frac{\partial V}{\partial x}\{A x+B v(\beta)\}\right\} \\
& =\max _{\beta \in \Delta(x)}\left\{\frac{\partial V}{\partial x}\{A x+B v(\beta)\}\right\} .
\end{aligned}
$$

Thus, in order to show (55), it is sufficient to prove that we have

$$
\max _{\beta \in \Delta(x)}\left\{\frac{\partial V}{\partial x}\{A x+B v(\beta)\}\right\}<0, \forall x \in \mathscr{L}_{V}\left(r^{-1}\right) \backslash\{0\} .
$$

The LMI (50) is equivalent to

$$
z^{T}\left[\begin{array}{cc}
A_{c l}^{T} P+P A_{c l} & P B-\Upsilon^{T}-A_{c l}^{T} H^{T} \Omega \\
B^{T} P-\Upsilon-\Omega H A_{c l} & -2 M-\Omega H B-(\Omega H B)^{T}
\end{array}\right] z<0
$$

for all $z \in \mathbb{R}^{n+m} \backslash\{0\}$.

Considering the vector $z^{T}=\left[x^{T} \phi(H x)^{T}\right]$ and $G=M^{-1} \Upsilon$, inequality (62) leads to

$$
\begin{aligned}
& x^{T}\left((A+B H)^{T} P+P(A+B H)\right) x \\
& +\phi(H x)^{T}\left(B^{T} P-M G-\Omega H(A+B H)\right) x \\
& +x^{T}\left(P B-G^{T} M^{T}-(A+B H)^{T} H^{T} \Omega\right) \phi(H x) \\
& +\phi(H x)^{T}\left(-2 M-\Omega H B-B^{T} H^{T} \Omega\right) \phi(H x) \\
& <0, \forall x \in \mathbb{R}^{n} \backslash\{0\} .
\end{aligned}
$$

Let us consider the notation $k(x)=H x+\phi(H x)$. According to Lemma 3 , for any $x \in \mathbb{R}^{n}$, we have $k(x) \in \mathscr{P}(c) \subseteq$ 
$\operatorname{Conv}\{\mathscr{V}\}$. Therefore, there exist $N$ positive scalars $\rho_{j}(x)$, $\sum_{j=1}^{N} \rho_{j}(x)=1$ such that

$$
k(x)=H x+\phi(H x)=\sum_{j=1}^{N} \rho_{j}(x) v_{j} .
$$

Using this property in (63), we obtain

$$
\begin{aligned}
& 2 x^{T} P\left(A x+B \sum_{j=1}^{N} \rho_{j}(x) v_{j}\right) \\
& -2 \phi(H x)^{T} \Omega H\left(A x+B \sum_{j=1}^{N} \rho_{j}(x) v_{j}\right) \\
& -2 \phi(H x)^{T} M(\phi(H x)+G x)<0, \forall x \in \mathbb{R}^{n} \backslash\{0\} .
\end{aligned}
$$

From (56), for any $x \in \mathbb{R}^{n}$ and $i \in \mathscr{I}(x)$ we have

$$
\left(x^{T} P-\phi(H x)^{T} \Omega H\right) B\left(v_{j}-v_{i}\right) \geq 0, \forall j \in \mathscr{I}_{N} .
$$

Then, for any $\beta \in \Delta(x)$ we get

$$
\left(x^{T} P-\phi(H x)^{T} \Omega H\right) B\left(v_{j}-v(\beta)\right) \geq 0, \forall j \in \mathscr{I}_{N} .
$$

By adding and subtracting the term $2 \sum_{j=1}^{N} \rho_{j}(x)\left(x^{T} P-\right.$ $\left.\phi(H x)^{T} \Omega H\right) B\left(v_{j}-v(\beta)\right)$ to (65), we obtain

$$
\begin{aligned}
& 2 x^{T} P(A x+B v(\beta)) \\
& -2 \phi(H x)^{T} \Omega H(A x+B v(\beta)) \\
& -2 \phi(H x)^{T} M(\phi(H x)+G x) \\
& +2 \sum_{j=1}^{N} \rho_{j}(x)\left(x^{T} P-\phi(H x)^{T} \Omega H\right) B\left(v_{j}-v(\beta)\right) \\
& <0, \forall x \in \mathbb{R}^{n} \backslash\{0\} .
\end{aligned}
$$

Applying Lemma 1, with $w_{1}=H x$ and $w_{2}=G x$, and using the definition of $\mathscr{P}(c)$ in (7), we have

$$
\phi(H x)^{T} M(\phi(H x)+G x) \leq 0, \forall x \in \mathscr{A},
$$

with

$$
\mathscr{A}=\left\{x \in \mathbb{R}^{n}:\left|\left(H_{(i)}-G_{(i)}\right) x\right| \leq c_{(i)}, \forall i \in \mathscr{I}_{m}\right\} .
$$

Note that $\frac{\partial V}{\partial x}=2 x^{T} P-2 \phi(H x)^{T} \Omega H$. Therefore, taking this into account, as well as (67) and (68), we deduce that

$$
\begin{aligned}
& \max _{\beta \in \Delta(x)}\left\{\frac{\partial V}{\partial x}(A x+B v(\beta))\right\} \\
& \leq \max _{\beta \in \Delta(x)}\left\{\frac{\partial V}{\partial x}(A x+B v(\beta))\right\}-2 \phi(H x)^{T} M(\phi(H x)+G x) \\
& +2 \sum_{j=1}^{N} \rho_{j}(x)\left(x^{T} P-\phi(H x)^{T} \Omega H\right) B\left(v_{j}-v(\beta)\right) \\
& <0, \forall x \in \mathscr{A} \backslash\{0\} .
\end{aligned}
$$

In order to show (61) (and thus (55)), we will now prove that $\mathscr{L}_{V}\left(r^{-1}\right) \subseteq \mathscr{A}$.

By multiplying (51) from both sides by $\left[\begin{array}{cc}I & 0 \\ 0 & \left(M_{(i, i)}\right)^{-1}\end{array}\right]$ and considering again $G=M^{-1} \Upsilon$, we obtain

$$
\left[\begin{array}{cc}
P & H_{(i)}^{T}-G_{(i)}^{T} \\
H_{(i)}-G_{(i)} & \frac{\tau_{(i)}}{M_{(i, i)}^{2}} c_{(i)}^{2}
\end{array}\right] \succeq 0, \forall i \in \mathscr{I}_{m} .
$$

Considering a scalar $r \geq \max _{i \in \mathscr{I}_{m}}\left\{\frac{\tau_{(i)}}{M_{(i, i)}^{2}}\right\}>0$, from (72), we obtain

$$
\left[\begin{array}{cc}
P & H_{(i)}^{T}-G_{(i)}^{T} \\
H_{(i)}-G_{(i)} & r c_{(i)}^{2}
\end{array}\right] \succeq 0, \forall i \in \mathscr{I}_{m} .
$$

This last inequality leads to

$$
x^{T}\left(H_{(i)}-G_{(i)}\right)^{T}\left(c_{(i)}^{2}\right)^{-1}\left(H_{(i)}-G_{(i)}\right) x \leq x^{T} \frac{P}{r^{-1}} x,
$$

for all $x \in \mathbb{R}^{n}$ and for all $i \in \mathscr{I}_{m}$.

This means in particular that for any $x \in \mathscr{E}\left(P, r^{-1}\right)$, one has

$$
x^{T}\left(H_{(i)}-G_{(i)}\right)^{T}\left(c_{(i)}^{2}\right)^{-1}\left(H_{(i)}-G_{(i)}\right) x \leq 1, \forall i \in \mathscr{I}_{m},
$$

which is equivalent to

$$
\left|\left(H_{(i)}-G_{(i)}\right) x\right| \leq c_{(i)}, \forall i \in \mathscr{I}_{m},
$$

and thus $x \in \mathscr{A}$ according to (70).

Therefore, equation (74) leads to the inclusion

$$
\mathscr{E}\left(P, r^{-1}\right) \subseteq \mathscr{A} .
$$

Applying Lemma 2, we also have

$$
x^{T} P x \leq V(x) \leq x^{T}\left(P+H^{T} \Omega H\right) x,
$$

which leads to the double inclusion

$$
\mathscr{E}\left(P+H^{T} \Omega H, r^{-1}\right) \subseteq \mathscr{L}_{V}\left(r^{-1}\right) \subseteq \mathscr{E}\left(P, r^{-1}\right) .
$$

Thus, from (79) and (77) we obtain

$$
\mathscr{L}_{V}\left(r^{-1}\right) \subseteq \mathscr{A} .
$$

Therefore, using (80) and (71), we have shown that (61) holds, and thus (55) is verified, which ends the proof. 
Remark 1 Note that inequalities (50) and (51) are affine in the matrix $A$. Then, the approach can be directly extended to the case of LTV systems with A varying in a convex polytope. In this case the condition should only be checked on the vertices of the polytope.

\subsection{Illustrative example}

In order to illustrate the performance of the proposed control method, we consider the linear system (4) with matrices

$A=\left[\begin{array}{ll}0 & 1 \\ 1 & 0\end{array}\right]$ and $B=\left[\begin{array}{cc}0 & 1 \\ -1 & 0.5\end{array}\right]$, and the controller $u$ which takes values in the set

$\mathscr{V}=\left\{\left[\begin{array}{c}25 \\ 25\end{array}\right],\left[\begin{array}{c}25 \\ -25\end{array}\right],\left[\begin{array}{c}-25 \\ 25\end{array}\right],\left[\begin{array}{l}-25 \\ -25\end{array}\right]\right\}$. One can verify that the open-loop linear system is unstable (the eigenvalues of the matrix $A$ are -1 and 1). Choosing a decay rate $\alpha=2.5$, we design the linear switching law proposed in [25] in order to stabilize the system to the origin. We obtain the following solutions of (20)

$$
Q=\left[\begin{array}{ll}
0.101 & 0.073 \\
0.073 & 0.172
\end{array}\right]
$$

and $\chi=1.6$. We deduce then

$$
H=\left[\begin{array}{ll}
-4.5 & 6.2 \\
-8.4 & 1.4
\end{array}\right]
$$

with an estimation of the ellipsoidal domain of attraction $\mathscr{E}(P, \gamma)$ where $\gamma=109.2$ and $P=Q^{-1}$. Based on Theorem 2 , we design a nonlinear switching law by solving LMIs (50) and (51) for $P=Q^{-1}$ and $H$ as given in (81)-(82) and a vector $c$ satisfying (7) such that $c_{(1)}=c_{(2)}=25$. We obtain $\Omega=\left[\begin{array}{cc}0.9 & 0 \\ 0 & 4.34\end{array}\right]$ and $r^{-1}=2.05 \times 10^{3}$. As we can see from

Figure 1, the obtained trajectories starting in the domain of attraction $\mathscr{L}_{V}\left(r^{-1}\right)$ converge to the origin. We can also note that, the domain of attraction $\mathscr{L}_{V}\left(r^{-1}\right)$ is larger than the ellipsoidal domain of attraction $\mathscr{E}(P, \gamma)$ obtained by the method proposed in [25]. The nonlinear switching surfaces and the convex hull $\mathscr{C}_{v}(H)$ defined in (21), which limits the domain of attraction in the approach in [25], are equally represented.

The evolution of the system's state starting from initial condition $x(0)=[6,6.2]^{T}$ (corresponding to the top right red star point in Figure 1) is presented in Figure 2, along with the evolution of the switching law's mode. This figure highlights both simple mode switching (at $t \simeq 0.06$ ) and sliding

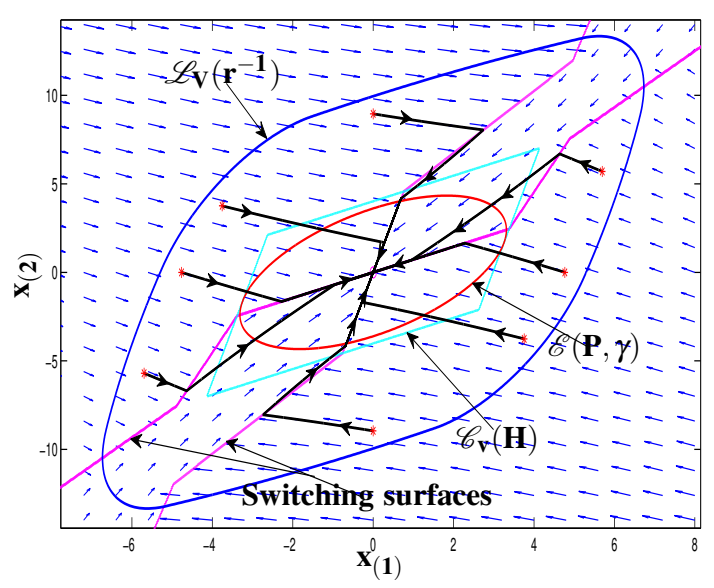

Fig. 1. Phase plot
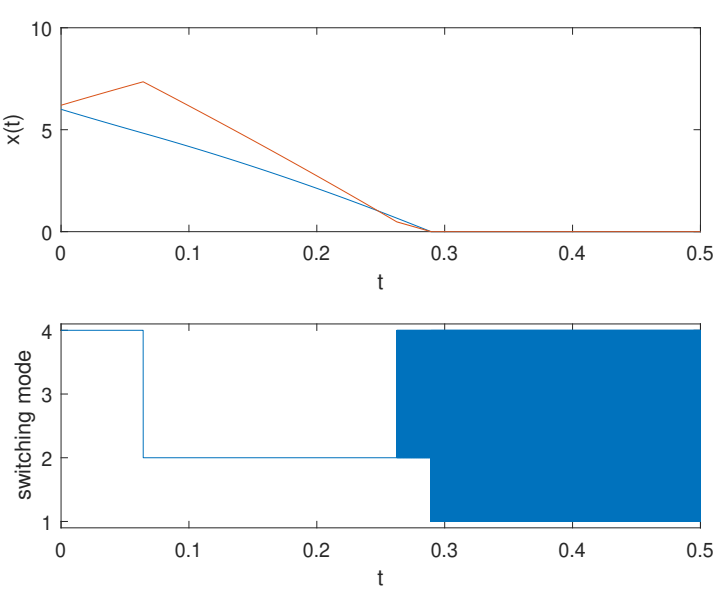

Fig. 2. Evolution of the system's state (top), and evolution of the switching law's mode (bottom), for initial condition $x(0)=[6,6.2]^{T}$

mode (starting at $t \simeq 0.26$ ), which can also be seen in Figure 1.

Assume now that the state matrix is affected by polytopic uncertainties :

$$
A(t) \in \operatorname{Conv}\left\{A_{1}, A_{2}\right\}, \forall t \geq 0,
$$

with $A_{1}=\left[\begin{array}{cc}0 & 1.5 \\ 1 & 0\end{array}\right]$ and $A_{2}=\left[\begin{array}{cc}0 & 1 \\ 1 & 0.5\end{array}\right]$

First, considering $\alpha=1.5$ and the method in [25], we solve the LMI (20) to obtain a common quadratic Lyapunov function for both subsystems 1 and 2. We obtain the following parameters of the linear switching law : $Q=\left[\begin{array}{ll}8.66 & 0.22 \\ 0.22 & 10.8\end{array}\right]$, 
$\chi=54.25$, and $H=\left[\begin{array}{cc}-0.064 & 2.52 \\ -3.1 & -1.194\end{array}\right]$. An estimation of the ellipsoidal domain of attraction is given by $\mathscr{E}\left(Q^{-1}, \gamma\right)$ with $\gamma=6.23$.

Next we solve the LMIs (50) and (51) for the same matrices $P=Q^{-1}, H$ and $c_{(1)}=c_{(2)}=25$. Then, we compute a Lur'e Lyapunov function for the subsystems 1 and $2\left(A_{1}\right.$ and $\left.A_{2}\right)$. We design the nonlinear switching law (52) with

$$
\Omega=\left[\begin{array}{cc}
0.11 & 0 \\
0 & 0.18
\end{array}\right]
$$

An estimation of the domain of attraction (53) is obtained with $r^{-1}=42.72$. For our simulations we consider

$$
\begin{aligned}
A(t) & =\left(\frac{\sin \left(x_{(1)}(t)+x_{(2)}(t)\right)+1}{2}\right) A_{1} \\
& +\left(1-\frac{\sin \left(x_{(1)}(t)+x_{(2)}(t)\right)+1}{2}\right) A_{2}
\end{aligned}
$$

Figure 3 shows the trajectories of the closed-loop system in the phase plot for different initial conditions together with the non-ellipsoidal domain of attraction $\mathscr{L}_{V}\left(r^{-1}\right)$ including the domain $\mathscr{E}(P, \gamma)$ obtained with the method proposed in [25].

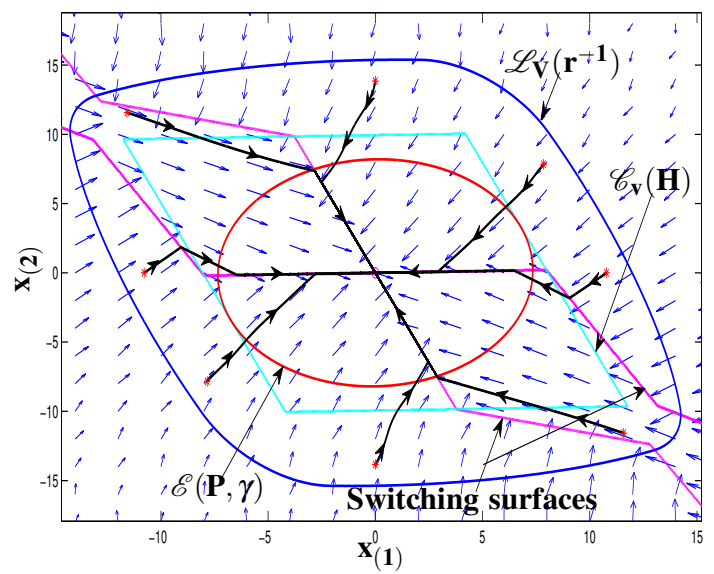

Fig. 3. Phase plot

We have proposed a constructive LMI-based method to design nonlinear state-dependent switching laws stabilizing the LTI system to the origin when the state variables are fully available to measurements. A larger non-ellipsoidal estimation of the domain of attraction has also been provided.

The following will now focus on the case where the system's state is not fully available to measurements.

\section{Observer-based relay feedback control design for LTI systems}

In this section, we consider the case where only a part of the state is measured. The output is defined as

$$
y=C x \text {. }
$$

For linear systems with continuous controller, it has been proved in the literature that the separation principle holds [43]. This result can not be applied to relay systems due to the discontinuous nature of the control. In this section, we provide a separation principle to relay systems. We also provide a constructive method based on LMIs for the design of an observer-based relay controller. Both linear and nonlinear switching surfaces dependent on the estimated state are designed. Using quadratic and non-quadratic Lyapunov functions, we also provide estimations of the domain of attraction.

We assume that A-1 and A-2 hold and also

A-3 The pair $(A, C)$ is detectable. This means that there exists a matrix $L$ such that $A_{o}=A+L C$ is Hurwitz.

In this case we provide a method for the stabilization of system (4) by an observer based relay feedback controller given by

$$
u(\hat{x}) \in \underset{v \in \mathscr{V}}{\arg \min } \Gamma(\hat{x}, v) .
$$

The estimated state $\hat{x} \in \mathbb{R}^{n}$ is computed by the full-order Luenberger state observer [36], [37]

$$
\left\{\begin{array}{l}
\dot{\hat{x}}=A \hat{x}+B u+L(\hat{y}-y) \\
\hat{y}=C \hat{x}
\end{array}\right.
$$

Our objective is to provide conditions which guarantee the existence of a mapping $\Gamma(\hat{x}, v)$ (which characterizes the switching surfaces of the control law) and a matrix $L$ (the observer gain) as well as a constructive method to design an observer-based controller such that the closed-loop system

$$
\left[\begin{array}{l}
\dot{x} \\
\dot{\hat{x}}
\end{array}\right]=\left[\begin{array}{cc}
A & 0 \\
L C & A+L C
\end{array}\right]\left[\begin{array}{l}
x \\
\hat{x}
\end{array}\right]+\left[\begin{array}{l}
B \\
B
\end{array}\right] u
$$

with the control law (85) is locally asymptotically stable.

Using the augmented state

$$
\xi=\left[\begin{array}{l}
\hat{x} \\
e
\end{array}\right]=\left[\begin{array}{cc}
0 & I \\
-I & I
\end{array}\right]\left[\begin{array}{l}
x \\
\hat{x}
\end{array}\right]
$$

where $e=\hat{x}-x$ is the estimation error, the interconnection 
(4), (86) can be written as the augmented closed-loop system

$$
\left\{\begin{array}{l}
\dot{\xi}=\left[\begin{array}{ll}
A & L C \\
0 & A+L C
\end{array}\right] \xi+\left[\begin{array}{l}
B \\
0
\end{array}\right] u(\hat{x}), \\
y=[C-C] \xi
\end{array}\right.
$$

which leads to

$$
\left\{\begin{array}{l}
\dot{\xi}=\tilde{A} \xi+\tilde{B} \bar{u}(\xi)=\tilde{\mathscr{X}}(\xi) \\
y=[C-C] \xi
\end{array}\right.
$$

where $\tilde{A}=\left[\begin{array}{cc}A & L C \\ 0 & A+L C\end{array}\right], \tilde{B}=\left[\begin{array}{l}B \\ 0\end{array}\right]$, and

$$
\bar{u}(\xi)=u\left(\left[\begin{array}{ll}
I & 0
\end{array}\right] \xi\right)=u(\hat{x}) \in \underset{v \in \mathscr{V}}{\arg \min } \Gamma\left(\left[\begin{array}{ll}
I & 0
\end{array}\right] \xi, v\right)
$$

In a similar way as in the full state feedback case we associate a differential inclusion

$$
\dot{\xi} \in \mathscr{F}[\tilde{\mathscr{X}}](\xi)
$$

to the system $(90),(91)$ with the set valued map $\mathscr{F}[\tilde{\mathscr{X}}](\xi)$ designed as

$$
\mathscr{F}[\tilde{\mathscr{X}}](\xi)=\bigcap_{\delta>0} \bigcap_{\mu(\mathscr{S})=0} \overline{\operatorname{Conv}}\{\tilde{\mathscr{X}}(\tilde{\mathscr{B}}(\xi, \delta)) \backslash \mathscr{S}\}, \forall \xi \in \mathbb{R}^{2 n}
$$

where $\tilde{\mathscr{B}}(\xi, \delta))$ is the open ball centred on $\xi$ with radius $\sqrt{\delta}$, and $\mathscr{S}$ is a set of measure (in the sense of Lebesgue) $\mu(\mathscr{S})=0$.

The problem considered in the previous section becomes:

Problem 2. Considering system (4), (84) and given a set $\mathscr{V}$, Assumptions A-1, A-2 and A-3, design an observer-based relay feedback controller such that the origin of the closedloop system is asymptotically stable.

In [30] we studied the existence of a mapping $\Gamma$ (characterizing the switching surface) and of an observer gain $L$ such that system (90), (91) (or equivalently (4), (84), (85), (86)) is locally exponentially stable. The result is reported in the following.

Theorem 3 [30] Assume that A.1, A.2, and A.3 hold. Then there exists a mapping $\Gamma(\hat{x}, v)=\hat{x}^{T} \Psi v$ (characterizing the switching hyperplanes) and a matrix $L$ (the observer gain) such that the origin of system (90), (91) (or equivalently of the closed-loop system (4), (84), (85), (86)) is locally exponentially stable.
This result has a qualitative nature. Here we are interested in finding a constructive procedure providing a mapping $\Gamma$ and an observer gain $L$ such that the origin of the closedloop system (90), (91) (or equivalently (4),(84), (85), (86)) is locally exponentially stable. We would also like to provide an estimation of the domain of attraction. In what follows, a numerical approach to deal with the design problem is given. An LMI solution is proposed hereafter.

For $\mathscr{H} \in \mathbb{R}^{m \times 2 n}$, let us define the set $\mathscr{C}_{v}(\mathscr{H})$ as follows

$$
\mathscr{C}_{v}(\mathscr{H})=\left\{\xi \in \mathbb{R}^{2 n}: l_{i} \mathscr{H} \xi \leq 1, \forall i \in \mathscr{I}_{n_{l}}\right\},
$$

where $l_{i}$ is given in (5).

Theorem 4 Assume that A.1, A.2, and A.3 hold. Consider a tuning parameter $\alpha>0$.

(1) If there exist positive definite matrices $Q_{1} \in \mathbb{R}^{n \times n}$ and $P_{2} \in \mathbb{R}^{n \times n}$, and scalars $\theta_{1}>0$ and $\theta_{2}>0$ such that

$$
\begin{gathered}
Q_{1} A^{T}+A Q_{1}-\theta_{1} B B^{T} \preceq-2 \alpha Q_{1}, \\
A^{T} P_{2}+P_{2} A-\theta_{2} C^{T} C \preceq-2 \alpha P_{2},
\end{gathered}
$$

then, the origin of system (4), (84), (86) with the switching law

$$
u(\hat{x}) \in \underset{v \in \mathscr{V}}{\arg \min } \hat{x}^{T} \Psi v,
$$

is locally $\alpha$-stable with $\Psi=Q_{1}^{-1} B$ and $L=-\frac{\theta_{2}}{2} P_{2}^{-1} C^{T}$.

(2) If in addition we consider $\lambda>0$ such that

$$
\left[\begin{array}{cc}
A_{c l}^{T} P_{1}+P_{1} A_{c l}+2 \alpha P_{1} & P_{1} L C \\
* & \lambda\left(A_{o}^{T} P_{2}+P_{2} A_{o}+2 \alpha P_{2}\right)
\end{array}\right] \preceq 0,
$$

with $A_{c l}=A-\frac{\theta_{1}}{2} B B^{T} Q_{1}^{-1}$ and $A_{o}=A+L C$, then an estimation of the domain of attraction is given by $\mathscr{E}(P, \bar{\gamma})$ with $P=\left[\begin{array}{cc}Q_{1}^{-1} & 0 \\ 0 & \lambda P_{2}\end{array}\right]$,

$$
\bar{\gamma} \leq \min _{i \in \mathscr{I}_{n_{l}}}\left(l_{i} \mathscr{H} P^{-1} \mathscr{H}^{T} l_{i}^{T}\right)^{-1}
$$

and $\mathscr{H}=\left[\begin{array}{ll}H & 0\end{array}\right]$ with $H=-\frac{\theta_{1}}{2} B^{T} Q_{1}^{-1}$.

(3) If there exist positive definite matrices $P_{1} \in \mathbb{R}^{n \times n}, Q_{2} \in$ $\mathbb{R}^{n \times n}$, and positive scalars $\theta_{1}$ and $\theta_{2}$ such that the LMIs (95), (96) are feasible for some $\alpha>0$, and if there exist two symmetric positive definite matrices $\tilde{P}_{1} \in \mathbb{R}^{n \times n}$ and $\tilde{P}_{2} \in \mathbb{R}^{n \times n}$, two diagonal positive definite matrices $\tilde{\Omega} \in$ $\mathbb{R}^{m \times m}$ and $\tilde{M} \in \mathbb{R}^{m \times m}$, a matrix $\tilde{\Upsilon} \in \mathbb{R}^{m \times n}$ and a strictly positive vector $\tilde{\tau} \in \mathbb{R}^{m}$ such that

$$
\left[\begin{array}{ccc}
A_{c l}^{T} \tilde{P}_{1}+\tilde{P}_{1} A_{c l} & \tilde{P}_{1} L C & \tilde{P}_{1} B-\tilde{\Upsilon}^{T}-A_{c l}^{T} H^{T} \tilde{\Omega} \\
* & A_{o}^{T} \tilde{P}_{2}+\tilde{P}_{2} A_{o} & -C^{T} L^{T} H^{T} \tilde{\Omega} \\
* & * & -2 \tilde{M}-\tilde{\Omega} H B-(\tilde{\Omega} H B)^{T}
\end{array}\right] \prec 0
$$


and

$$
\left[\begin{array}{cc}
\tilde{P}_{1} & \tilde{M}_{(i, i)} H_{(i)}^{T}-\tilde{\Upsilon}_{(i)}^{T} \\
* & \tilde{\tau}_{(i)} c_{(i)}^{2}
\end{array}\right] \succeq 0, \forall i \in \mathscr{I}_{m}
$$

with $L=-\frac{\theta_{2}}{2} P_{2}^{-1} C^{T}, A_{c l}=A-\frac{\theta_{1}}{2} B B^{T} Q_{1}^{-1}, \quad H=$ $-\frac{\theta_{1}}{2} B^{T} Q_{1}^{-1}$, and $A_{o}=A+L C$, then the origin of system (4), (84), (86) with the switching law

$$
u(\hat{x}) \in \underset{v \in \mathscr{V}}{\arg \min }\left(\hat{x}^{T} \tilde{P}_{1}-\phi(H \hat{x})^{T} \tilde{\Omega} H\right) B v
$$

is locally asymptotically stable.

An estimation of the domain of attraction is given by

$$
\mathscr{L}_{V}\left(\eta^{-1}\right)=\left\{\xi \in \mathbb{R}^{2 n}: V(\xi) \leq \eta^{-1}\right\},
$$

with

$$
\begin{gathered}
V(\xi)=\xi^{T} \tilde{P} \xi-2 \sum_{j=1}^{m} \int_{0}^{\mathscr{H}_{(j)} \xi} \phi_{(j)}(\sigma) \tilde{\Omega}_{(j, j)} d \sigma \\
\xi=\left[\begin{array}{l}
\hat{x} \\
e
\end{array}\right], \tilde{P}=\left[\begin{array}{cc}
\tilde{P}_{1} & 0 \\
0 & \tilde{P}_{2}
\end{array}\right] \text {, and } \eta \geq \max _{i \in \mathscr{I}_{m}}\left\{\frac{\tilde{\tau}_{(i)}}{\tilde{M}_{(i, i)}^{2}}\right\} .
\end{gathered}
$$

Proof. 1. Consider positive definite matrices $Q_{1}, P_{2}$, and positive scalars $\theta_{1}, \theta_{2}$ such that (95), (96) hold. Then, we want to prove that the origin of the closed-loop system (90), (97), with $\Psi=Q_{1}^{-1} B$ and $L=-\frac{\theta_{2}}{2} P_{2}^{-1} C^{T}$, is locally $\alpha$-stable in some domain $\mathscr{D} \subset \mathbb{R}^{2 n}$.

We can remark that the feasibility of (95) implies that the inequality

$$
A_{c l}^{T} P_{1}+P_{1} A_{c l} \preceq-2 \alpha P_{1}
$$

is verified with $P_{1}=Q_{1}^{-1}$, and $A_{c l}=A-\frac{\theta_{1}}{2} B B^{T} Q_{1}^{-1}$ (see for instance [10]).

Similarly, the feasibility of LMI (96) implies that the inequality

$$
A_{o}^{T} P_{2}+P_{2} A_{o} \preceq-2 \alpha P_{2}
$$

is verified with $L=-\frac{\theta_{2}}{2} P_{2}^{-1} C^{T}$ and $A_{o}=A+L C$. Let us consider the quadratic Lyapunov function $V(\xi)=\xi^{T} P \xi$, with $P=\left[\begin{array}{cc}Q_{1}^{-1} & 0 \\ 0 & \lambda P_{2}\end{array}\right]$ and $\lambda>0$.

Recall the differential inclusion (92) associated to the closedloop system (90), (91). In order to show the local $\alpha$-stability of the closed-loop system at the origin, it is sufficient to prove that

$$
\sup _{\varsigma \in \mathscr{F}[\tilde{\mathscr{X}}](\xi)} \frac{\partial V}{\partial \xi} \varsigma \leq-2 \alpha V(\xi),
$$

in some domain $\mathscr{D}$ around the origin.
First let us define for each $\hat{x} \in \mathbb{R}^{n}$ the set of minimizers in which the controller (97) takes values. Since we have

$$
\hat{x}^{T} \Psi v=\hat{x}^{T} P_{1} B v=\xi^{T}\left[\begin{array}{l}
I \\
0
\end{array}\right]\left[\begin{array}{ll}
I & 0
\end{array}\right] P \tilde{B} v,
$$

and since

$$
\xi^{T}\left[\begin{array}{l}
I \\
0
\end{array}\right]\left[\begin{array}{ll}
I & 0
\end{array}\right] P \tilde{B} v=\xi^{T} P \tilde{B} v
$$

is satisfied thanks to the particular structure of $\tilde{B}$ and $P$, this set of minimizers can be defined for all $z \in \mathbb{R}^{2 n}$ as follows

$$
\mathscr{I}(z)=\left\{i \in \mathscr{I}_{N}: z^{T} P \tilde{B}\left(v_{j}-v_{i}\right) \geq 0, \forall j \in \mathscr{I}_{N}\right\}
$$

To this set of indexes, we associate the set $\Delta(z)$ of vectors defined for all $z \in \mathbb{R}^{2 n}$ by

$$
\Delta(z)=\left\{\beta \in \Delta_{N}: \beta_{(i)}=0, \forall i \in \mathscr{I}_{N} \backslash \mathscr{I}(z)\right\}
$$

Using (110) and (111), the set valued map $\mathscr{F}[\tilde{\mathscr{X}}](\xi)$ in (92) satisfies

$$
\mathscr{F}[\tilde{\mathscr{X}}](\xi) \subseteq \tilde{\mathscr{F}}[\tilde{\mathscr{X}}](\xi)
$$

with

$$
\begin{aligned}
\tilde{\mathscr{F}}[\tilde{\mathscr{X}}](\xi) & =\operatorname{Conv}\left\{\tilde{A} \xi+\tilde{B} v_{i}: i \in \mathscr{I}(\xi)\right\} \\
& =\{\tilde{A} \xi+\tilde{B} v(\beta): \beta \in \Delta(\xi)\}
\end{aligned}
$$

where $v(\beta)=\sum_{i=1}^{N} \beta_{(i)} v_{i}$.

From (112) and (113) and using the fact that $\Delta(\xi)$ is compact, we have

$$
\begin{aligned}
\sup _{\varsigma \in \tilde{F}[\tilde{\mathscr{X}}](\xi)} \frac{\partial V}{\partial \xi} \varsigma & \leq \sup _{\varsigma \in \tilde{\mathscr{F}}[\tilde{\mathscr{X}}](\xi)} \frac{\partial V}{\partial \xi} \varsigma \\
& =\sup _{\beta \in \Delta(\xi)}\left\{\frac{\partial V}{\partial \xi}\{\tilde{A} \xi+\tilde{B} v(\beta)\}\right\} \\
& =\max _{\beta \in \Delta(\xi)}\left\{\frac{\partial V}{\partial \xi}\{\tilde{A} \xi+\tilde{B} v(\beta)\}\right\} .
\end{aligned}
$$

Thus, in order to show (107), it is sufficient to prove that we have

$$
\max _{\beta \in \Delta(\xi)}\left\{\frac{\partial V}{\partial \xi}\{\tilde{A} \xi+\tilde{B} v(\beta)\}\right\} \leq-2 \alpha V(\xi),
$$

in some domain $\mathscr{D}$ around the origin.

Note that, since A-2 holds, then there exists a neighbourhood of the origin $\mathscr{E}(P, \gamma) \subset \mathbb{R}^{2 n}$, with $\gamma>0$ such that for all $\xi \in \mathscr{E}(P, \gamma)$, we have

$$
\mathscr{H} \xi \in \operatorname{Conv}\{\mathscr{V}\}
$$


with $\mathscr{H}=\left[\begin{array}{ll}H & 0\end{array}\right]$.

Therefore, for all $\xi \in \mathscr{E}(P, \gamma)$ there exist scalars $\rho_{j}(\xi), j \in$ $\mathscr{I}_{N}$ such that $\sum_{j=1}^{N} \rho_{j}(\xi)=1$ and

$$
\mathscr{H} \xi=\sum_{j=1}^{N} \rho_{j}(\xi) v_{j}
$$

From (110), for all $i \in \mathscr{I}(\xi)$ we have

$$
\xi^{T} P \tilde{B}\left(v_{j}-v_{i}\right) \geq 0, \forall j \in \mathscr{I}_{N}
$$

Then, for any $\beta \in \Delta(\xi)$, we have

$$
\xi^{T} P \tilde{B}\left(v_{j}-v(\beta)\right) \geq 0, \forall j \in \mathscr{I}_{N}
$$

Then, considering (117), and multiplying the last inequalities by $\rho_{j}(\xi)$ and summing the $N$ elements we obtain

$$
\xi^{T} P \tilde{B}(\mathscr{H} \xi-v(\beta)) \geq 0
$$

Adding this to the left part of (115), it comes

$$
\begin{aligned}
& \max _{\beta \in \Delta(\xi)}\left\{\frac{\partial V}{\partial \xi}\{\tilde{A} \xi+\tilde{B} v(\beta)\}\right\} \\
& \leq \max _{\beta \in \Delta(\xi)}\left\{2 \xi^{T} P(\tilde{A} \xi+\tilde{B} v(\beta))+2 \xi^{T} P \tilde{B}(\mathscr{H} \xi-v(\beta))\right\} \\
& =2 \xi^{T} P(\tilde{A} \xi+\tilde{B} \mathscr{H} \xi)=2 \xi^{T} P\left[\begin{array}{cc}
A_{c l} & L C \\
0 & A_{o}
\end{array}\right] \xi:=2 \xi^{T} P \tilde{A}_{c l} \xi
\end{aligned}
$$

for all $\xi \in \mathscr{E}(P, \gamma)$, with $A_{c l}=A+B H$ and $A_{o}=A+L C$.

Thus, in order to show (107), it is sufficient to prove that

$$
2 \xi^{T} P \tilde{A}_{c l} \xi \leq-2 \alpha V(\xi)=-2 \alpha \xi^{T} P \xi, \forall \xi \in \mathscr{E}(P, \gamma)
$$

This last inequality holds if

$$
\tilde{A}_{c l}^{T} P+P \tilde{A}_{c l} \preceq-2 \alpha P
$$

is satisfied.

Note that

$$
\begin{aligned}
& \tilde{A}_{c l}^{T} P+P \tilde{A}_{c l}+2 \alpha P= \\
& {\left[\begin{array}{cc}
A_{c l}^{T} P_{1}+P_{1} A_{c l}+2 \alpha P_{1} & P_{1} L C \\
(L C)^{T} P_{1} & \lambda\left(A_{o}^{T} P_{2}+P_{2} A_{o}+2 \alpha P_{2}\right)
\end{array}\right]}
\end{aligned}
$$

Applying the Schur complement, the matrix (124) is negative if and only if

$$
A_{o}^{T} P_{2}+P_{2} A_{o}+2 \alpha P_{2} \preceq 0
$$

and

$$
\begin{aligned}
& \left(A_{c l}^{T} P_{1}+P_{1} A_{c l}+2 \alpha P_{1}\right) \\
& -\frac{1}{\lambda} P_{1} L C\left[2 \alpha P_{2}+A_{o}^{T} P_{2}+P_{2} A_{o}\right]^{-1}(L C)^{T} P_{1} \preceq 0 .
\end{aligned}
$$

Since (105) and (106) are satisfied, it is clear that if we take $\lambda$ large enough both inequalities (125) and (126) are verified. Therefore, we have shown that there exist a Lyapunov function $V$ such that (107) holds for any $\xi$ in a positive invariant set $\mathscr{E}(P, \gamma)$, and thus there exist a linear mapping $\Gamma(\hat{x}, v)=\hat{x}^{T} \Psi v=\hat{x}^{T} Q_{1}^{-1} B v$ and an observer gain $L=\frac{-\theta_{2}}{2} P_{2}^{-1} C^{T}$ such that the origin of system (90), (97) (and equivalently (4), (84), (97), (86)) is locally $\alpha$-stable in some domain of attraction $\mathscr{E}(P, \gamma)$.

2. It is now required to estimate the domain of attraction for system (90) with the switching law (97). Thus, we want to determine a scalar $\bar{\gamma}$ characterizing the ellipsoid $\mathscr{E}(P, \bar{\gamma})$ such that

$$
\sup _{\varsigma \in \tilde{F}[\tilde{\mathscr{Y}}](\xi)} \frac{\partial V}{\partial \xi} \varsigma \leq-2 \alpha V(\xi), \forall \xi \in \mathscr{E}(P, \bar{\gamma}) .
$$

For a given decay rate $\alpha$ and if LMIs (95) and (96) are feasible, then from the result above, there exist at least one scalar $\lambda$ satisfying the inequality (98).

Considering such a scalar $\lambda$, our objective here is to provide an estimation $\mathscr{E}(P, \bar{\gamma})$ of the domain of attraction such that

$$
\mathscr{E}(P, \bar{\gamma}) \subseteq \mathscr{C}_{v}(\mathscr{H})
$$

with $\mathscr{C}_{v}(\mathscr{H})$ defined in (94). Note that, if the set $\mathscr{E}(P, \bar{\gamma})$ satisfies (128), then according to (5), one will have that for all $\xi \in \mathscr{E}(P, \bar{\gamma}), \mathscr{H} \xi \in \operatorname{Conv}\{\mathscr{V}\}$, and the set will satisfy the condition (117) assumed in the first part of the proof.

For this inclusion to hold it is both necessary and sufficient that none of the hyperplanes $l_{i} \mathscr{H} \xi=1, i \in \mathscr{I}_{n_{l}}$ crosses the level set $\mathscr{E}(P, \bar{\gamma})$. Note that for any $i \in \mathscr{I}_{n_{l}}$, the minimum of $V$ along the hyperplane $\left\{\xi \in \mathbb{R}^{2 n}: l_{i} \mathscr{H} \xi=1\right\}$ is given as (see [10])

$$
\min _{l_{i} \mathscr{H} \xi=1} \xi^{T} P \xi=\min _{i \in \mathscr{I}_{n_{l}}}\left(l_{i} \mathscr{H} P^{-1} \mathscr{H}^{T} l_{i}^{T}\right)^{-1} .
$$

We can remark that by taking $\bar{\gamma}$ as

$$
\bar{\gamma} \leq \min _{l_{i} \mathscr{H} \xi=1} \xi^{T} P \xi=\left(l_{i} \mathscr{H} P^{-1} \mathscr{H}^{T} l_{i}^{T}\right)^{-1}
$$

the inclusion (128) is verified and $\mathscr{E}(P, \bar{\gamma})$ is thus an estimation of the domain of attraction.

3. The aim here is to show that if there exist positive definite matrices $Q_{1}, P_{2}$, and positive scalars $\theta_{1}$ and $\theta_{2}$ satisfying 
(95) and (96) and the LMIs (100) and (101) are feasible with $H=-\frac{\theta_{1}}{2} B^{T} Q_{1}^{-1}$ and $L=-\frac{\theta_{2}}{2} P_{2}^{-1} C^{T}$, then the origin of the closed-loop system (4), (84), (86), (102) is locally asymptotically stable in the domain of attraction $\mathscr{L}_{V}\left(\eta^{-1}\right)$.

Recall that we associate to the closed-loop system (90), (102) the differential inclusion (92), which is locally asymptotically stable at the origin in the domain $\mathscr{L}_{V}\left(\eta^{-1}\right)$ if

$$
\sup _{\varsigma \in \mathscr{F}[\tilde{\mathscr{X}}](\xi)} \frac{\partial V}{\partial \xi} \varsigma<0, \forall \xi \in \mathscr{L}_{V}\left(\eta^{-1}\right) \backslash\{0\} .
$$

Thanks to the particular structure of the matrices $\tilde{P}=$ $\left[\begin{array}{cc}\tilde{P}_{1} & 0 \\ 0 & \tilde{P}_{2}\end{array}\right], \mathscr{H}=\left[\begin{array}{ll}H & 0\end{array}\right]$, and $\tilde{B}=\left[\begin{array}{l}B \\ 0\end{array}\right]$, we can show that

$$
\left(\hat{x}^{T} \tilde{P}_{1}-\phi(H \hat{x})^{T} \tilde{\Omega} H\right) B v=\left(\xi^{T} \tilde{P}-\phi(\mathscr{H} \xi)^{T} \tilde{\Omega} \mathscr{H}\right) \tilde{B} v .
$$

Let us first define for all $\zeta \in \mathbb{R}^{2 n}$ the set of minimizers in which the controller (102) takes values:

$$
\begin{aligned}
& \mathscr{I}(\zeta)=\left\{i \in \mathscr{I}_{N}:\right. \\
& \left.\left(\zeta^{T} \tilde{P}-\phi(\mathscr{H} \zeta)^{T} \tilde{\Omega} \mathscr{H}\right) \tilde{B}\left(v_{j}-v_{i}\right) \geq 0, \forall j \in \mathscr{I}_{N}\right\} .
\end{aligned}
$$

To this set of indexes, we associate the set of vectors $\Delta(\zeta)$ defined for all $\zeta \in \mathbb{R}^{2 n}$ as

$$
\Delta(\zeta)=\left\{\beta \in \Delta_{N}: \beta_{(i)}=0, \forall i \in \mathscr{I}_{N} \backslash \mathscr{I}(\zeta)\right\}
$$

Using (133) and (134) we obtain that the set valued map $\mathscr{F}[\mathscr{X}](\xi)$ satisfies the following relation

$$
\mathscr{F}[\tilde{\mathscr{X}}](\xi) \subseteq \tilde{\mathscr{F}}[\tilde{\mathscr{X}}](\xi)
$$

with

$$
\begin{aligned}
\tilde{\mathscr{F}}[\tilde{\mathscr{X}}](\xi) & =\operatorname{Conv}\left\{\tilde{A} \xi+\tilde{B} v_{i}: i \in \mathscr{I}(\xi)\right\} \\
& =\{\tilde{A} \xi+\tilde{B} v(\beta): \beta \in \Delta(\xi)\}
\end{aligned}
$$

where $v(\beta)=\sum_{i=1}^{N} \beta_{(i)} v_{i}$. Thus, using the same argument as in the first part of proof we can show that to prove (131) it is sufficient to show that

$$
\max _{\beta \in \Delta(\zeta)}\left\{\frac{\partial V}{\partial \xi}(\tilde{A} \xi+\tilde{B} v(\beta))\right\}<0, \forall \xi \in \mathscr{L}_{V}\left(\eta^{-1}\right) \backslash\{0\}
$$

Considering the particular structure of matrices $\mathscr{H}=$ $\left[\begin{array}{ll}H & 0\end{array}\right], \tilde{P}=\left[\begin{array}{cc}\tilde{P}_{1} & 0 \\ 0 & \tilde{P}_{2}\end{array}\right], \tilde{B}=\left[\begin{array}{l}B \\ 0\end{array}\right]$, and $\tilde{\mathscr{Y}}=\left[\begin{array}{ll}\tilde{\Upsilon} & 0\end{array}\right]$, we can show that (100) is equivalent to

$$
\left[\begin{array}{cc}
\tilde{A}_{c l}^{T} \tilde{P}+\tilde{P} \tilde{A}_{c l} & \tilde{P} \tilde{B}-\tilde{\mathscr{Y}}^{T}-\tilde{A}_{c l}^{T} \mathscr{H}^{T} \tilde{\Omega} \\
* & -2 \tilde{M}-\tilde{\Omega} \mathscr{H} \tilde{B}-(\tilde{\Omega} \mathscr{H} \tilde{B})^{T}
\end{array}\right] \prec 0 .
$$

From (138) we have for all $\Xi \in \mathbb{R}^{2 n+m} \backslash\{0\}$

$$
\Xi^{T}\left[\begin{array}{cc}
\tilde{A}_{c l}^{T} \tilde{P}+\tilde{P} \tilde{A}_{c l} & \tilde{P} \tilde{B}-\tilde{\mathscr{Y}}^{T}-\tilde{A}_{c l}^{T} \mathscr{H}^{T} \tilde{\Omega} \\
* & -2 \tilde{M}-\tilde{\Omega} \mathscr{H} \tilde{B}-(\tilde{\Omega} \mathscr{H} \tilde{B})^{T}
\end{array}\right] \Xi<0 .
$$

Considering $\Xi=\left[\begin{array}{c}\xi \\ \phi(\mathscr{H} \xi)\end{array}\right]$ with $\xi \in \mathbb{R}^{2 n} \backslash\{0\}$ and $\mathscr{G}=$ $\tilde{M}^{-1} \tilde{\mathscr{Y}},(139)$ leads to

$$
\begin{aligned}
& \left(2 \xi^{T} \tilde{P}-2 \phi(H \xi)^{T} \tilde{\Omega} \mathscr{H}\right)((\tilde{A}+\tilde{B} \mathscr{H}) \xi+\tilde{B} \phi(\mathscr{H} \xi)) \\
& -2 \phi(\mathscr{H} \xi)^{T} \tilde{M}(\phi(\mathscr{H} \xi)+\mathscr{G} \xi) \\
& <0, \forall \xi \in \mathbb{R}^{2 n} \backslash\{0\} .
\end{aligned}
$$

Let us consider the notation $k(\xi)=\mathscr{H} \xi+\phi(\mathscr{H} \xi)$. According to Lemma 3 , for any $\xi \in \mathbb{R}^{n}$, we have $k(\xi) \in \mathscr{P}(c) \subseteq$ $\operatorname{Conv}\{\mathscr{V}\}$. Therefore, there exist $N$ positive scalars $\rho_{j}(\xi)$, $\sum_{j=1}^{N} \rho_{j}(\xi)=1$ such that

$$
k(\xi)=\mathscr{H} \xi+\phi(\mathscr{H} \xi)=\sum_{j=1}^{N} \rho_{j}(\xi) v_{j}
$$

Using this property in (140), we get

$$
\begin{aligned}
& \left(2 \xi^{T} \tilde{P}-2 \phi(\mathscr{H} \xi)^{T} \tilde{\Omega} \mathscr{H}\right)\left(\tilde{A} \xi+\tilde{B} \sum_{j=1}^{N} \rho_{j}(\xi) v_{j}\right) \\
& -2 \phi(\mathscr{H} \xi)^{T} \tilde{M}(\phi(\mathscr{H} \xi)+\mathscr{G} \xi) \\
& <0, \forall \xi \in \mathbb{R}^{2 n} \backslash\{0\} .
\end{aligned}
$$

From (133), for all $i \in \mathscr{I}(\xi)$ we have

$$
\left(\xi^{T} \tilde{P}-\phi(\mathscr{H} \xi)^{T} \tilde{\Omega} \mathscr{H}\right) \tilde{B}\left(v_{j}-v_{i}\right) \geq 0, \forall j \in \mathscr{I}_{N} .
$$

Thus, for all $\beta \in \Delta(\xi)$ we obtain

$$
\left(\xi^{T} \tilde{P}-\phi(\mathscr{H} \xi)^{T} \tilde{\Omega} \mathscr{H}\right) \tilde{B}\left(v_{j}-v(\beta)\right) \geq 0, \forall j \in \mathscr{I}_{N} .
$$

Therefore, by adding and subtracting the term

$2 \sum_{j=1}^{N} \rho_{j}(\xi)\left(\xi^{T} \tilde{P}-\phi(\mathscr{H} \xi)^{T} \tilde{\Omega} \mathscr{H}\right) \tilde{B}\left(v_{j}-v(\beta)\right)$ to (142), we obtain

$$
\begin{aligned}
& \left(2 \xi^{T} \tilde{P}-2 \phi(\mathscr{H} \xi)^{T} \tilde{\Omega} \mathscr{H}\right)(\tilde{A} \xi+\tilde{B} v(\beta)) \\
& -2 \phi(\mathscr{H} \xi)^{T} \tilde{M}(\phi(\mathscr{H} \xi)+\mathscr{G} \xi) \\
& +\sum_{j=1}^{N} \rho_{j}(\xi)\left(\xi \tilde{P}-\phi(\mathscr{H} \xi)^{T} \tilde{\Omega} \mathscr{H}\right)\left(v_{j}-v(\beta)\right) \\
& <0, \forall \xi \in \mathbb{R}^{2 n} \backslash\{0\} .
\end{aligned}
$$

Applying Lemma 1 with $w_{1}=\mathscr{H} \xi, w_{2}=\mathscr{G} \xi$ and using the definition of $\mathscr{P}(c)$ in (7), we have

$$
\phi(\mathscr{H} \xi)^{T} \tilde{M}(\phi(\mathscr{H} \xi)+\mathscr{G} \xi) \leq 0, \forall \xi \in \tilde{\mathscr{A}}
$$


with

$$
\tilde{\mathscr{A}}=\left\{\xi \in \mathbb{R}^{2 n}:\left|\left(\mathscr{H}_{(i)}-\mathscr{G}_{(i)}\right) \xi\right| \leq c_{(i)}, \forall i \in \mathscr{I}_{m}\right\} .
$$

Note that $\frac{\partial V}{\partial \xi}=2 \xi^{T} \tilde{P}-2 \phi(\mathscr{H} \xi)^{T} \tilde{\Omega} \mathscr{H}$. Therefore, taking this into account, as well as (145) and (146), we obtain

$$
\begin{aligned}
& \max _{\beta \in \Delta(\xi)}\left\{\frac{\partial V}{\partial \xi}(\tilde{A} \xi+\tilde{B} v(\beta))\right\} \\
& \leq \max _{\beta \in \Delta(\xi)}\left\{\frac{\partial V}{\partial \xi}(\tilde{A} \xi+\tilde{B} v(\beta))\right\} \\
& -2 \phi(\mathscr{H} \xi)^{T} \tilde{M}(\phi(\mathscr{H} \xi)+\mathscr{G} \xi) \\
& +\sum_{j=1}^{N} \rho_{j}(\xi)\left(\xi \tilde{P}-\phi(\mathscr{H} \xi)^{T} \tilde{\Omega} \mathscr{H}\right)\left(v_{j}-v(\beta)\right) \\
& <0, \forall \xi \in \mathscr{A} .
\end{aligned}
$$

In order to show (137) (and thus (131)), we will now prove that $\mathscr{L}_{V}\left(\eta^{-1}\right) \subseteq \tilde{A}$.

Considering the same arguments as in Theorem 2 we can show that (101) is equivalent to

$$
\left[\begin{array}{cc}
\tilde{P}_{1} & H_{(i)}^{T}-\tilde{G}_{(i)}^{T} \\
H_{(i)}-G_{(i)} & \eta c_{(i)}^{2}
\end{array}\right] \succeq 0, \forall i \in \mathscr{I}_{m}
$$

Applying the Schur complement to (149), we obtain

$$
\frac{\tilde{P}_{1}}{\eta^{-1}}-\left(H_{(i)}-\tilde{G}_{(i)}\right)^{T}\left(c_{(i)}^{2}\right)^{-1}\left(H_{(i)}-G_{(i)}\right) \succeq 0, \forall i \in \mathscr{I}_{m}
$$

Since $\tilde{P}_{2} \succ 0$ and $\eta>0$ then (150) leads to

$$
\left[\begin{array}{cc}
\frac{\tilde{P}_{1}}{\eta^{-1}}-\left(H_{(i)}-\tilde{G}_{(i)}\right)^{T}\left(c_{(i)}^{2}\right)^{-1}\left(H_{(i)}-G_{(i)}\right) & 0 \\
0 & \frac{\tilde{P}_{2}}{\eta^{-1}}
\end{array}\right] \succeq 0, \forall i \in \mathscr{I}_{m},
$$

which is equivalent to

$$
\left[\begin{array}{cc}
\frac{\tilde{P}_{1}}{\eta^{-1}} & 0 \\
0 & \frac{\tilde{P}_{2}}{\eta^{-1}}
\end{array}\right]-\left[\begin{array}{c}
\left(H_{(i)}-\tilde{G}_{(i)}\right)^{T} \\
0
\end{array}\right]\left(c_{(i)}^{2}\right)^{-1}\left[H_{(i)}-\tilde{G}_{(i)} 0\right] \succeq 0,
$$

for all $i \in \mathscr{I}_{m}$.

For all $\xi \in \mathbb{R}^{2 n}$ and $\mathscr{G}=\tilde{M}^{-1} \tilde{\mathscr{Y}}=\left[\begin{array}{ll}\tilde{G} & 0\end{array}\right]$, (152) leads to

$\xi^{T} \frac{\tilde{P}}{\eta^{-1}} \xi-\xi^{T}\left(\mathscr{H}_{(i)}-\mathscr{G}_{(i)}\right)^{T}\left(c_{(i)}^{2}\right)^{-1}\left(\mathscr{H}_{(i)}-\mathscr{G}_{(i)}\right) \xi \geq 0, \forall i \in \mathscr{I}_{m}$.
From this we obtain the inclusion

$$
\mathscr{E}\left(\tilde{P}, \eta^{-1}\right) \subseteq \tilde{\mathscr{A}}
$$

In addition, according to Lemma 2, we have

$$
\xi^{T} \tilde{P} \xi \leq V(\xi) \leq \xi^{T} \bar{P} \xi
$$

where $\bar{P}=\left[\begin{array}{cc}\tilde{P}_{1}+H^{T} \Omega H & 0 \\ 0 & \tilde{P}_{2}\end{array}\right]$.

This leads to

$$
\mathscr{E}\left(\bar{P}, \eta^{-1}\right) \subseteq \mathscr{L}_{V}\left(\eta^{-1}\right) \subseteq \mathscr{E}\left(\tilde{P}, \eta^{-1}\right)
$$

Thus, from (156) and (154), we have

$$
\mathscr{L}_{V}\left(\eta^{-1}\right) \subseteq \tilde{\mathscr{A}}
$$

From this and using (148) we have shown that (137) holds and we get

$$
\begin{aligned}
\sup _{\varsigma \in \tilde{F}[\tilde{\mathscr{X}}](\xi)} \frac{\partial V}{\partial \xi} \varsigma & \leq \max _{\beta \in \Delta(\xi)}\left\{\frac{\partial V}{\partial \xi}(\tilde{A} \xi+\tilde{B} v(\beta))\right\} \\
& <0, \forall \xi \in \mathscr{L}_{V}\left(\eta^{-1}\right) \backslash\{0\}
\end{aligned}
$$

which ends the proof.

Remark 2 We can observe that (95), (96) do not share cross variables, thus they can be solved separately. Therefore, the control law matrix $\Psi$ and the observer gain $L$ can be designed independently, which shows that the separation principle holds in the case of relay systems.

Remark 3 The feasibility of the set of conditions (95)-(96) allows the design of the matrix $\Psi$ for the switching law and the observer gain L separately, and equations (98) and (99) provide an estimation $\mathscr{E}(P, \bar{\gamma})$ of the domain of attraction such that any solution of the closed-loop system (90), (91) starting in the domain of attraction $\mathscr{E}(P, \bar{\gamma})$ converges to the origin exponentially with a decay rate $\alpha$.

The numerical implementation can be done in two steps. First, LMIs (95)-(96) are solved to find the matrices $P_{1}, \theta_{1}$, $P_{2}, \theta_{2}, L$, and $\Psi$. In the second step, $\lambda$ is computed from (98) and then the estimation of the domain of attraction can be computed using the equation (99). An optimization of the domain of attraction can be done by using recursive LMI algorithms.

In addition the feasibility of LMIs (100), (101) allows the design of nonlinear switching laws dependent on the estimated states ensuring the local asymptotic stability of the origin in a larger non-ellipsoidal domain of attraction. 


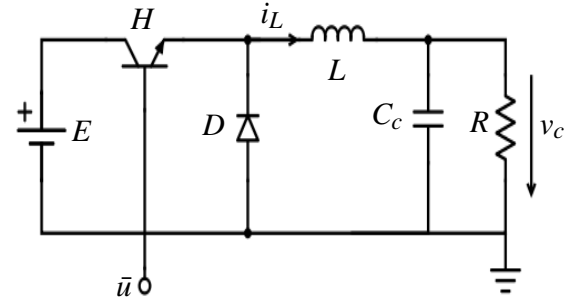

Fig. 4. Buck converter

Remark 4 The feasibility of the LMIs (95)-(96) is guaranteed for a sufficiently small $\alpha$ since Assumptions A.1 and A.3 hold. Thus, for a sufficiently small decay rate $\alpha$, since system (4) is stabilizable, there exist a gain $H$ such that $A+B H$ is Hurwitz and a symmetric positive definite matrix $P=Q^{-1}$ satisfying (95). Likewise, since system (4) is detectable then there exist a gain $L$ such that $A+L C$ is Hurwitz and a symmetric positive definite matrix $P_{2}$ verifying (96) for a sufficiently small decay rate $\alpha$.

\section{$4.1 \quad$ Numerical example: Buck converter}

Let us consider the buck converter [6] shown in Figure 4. The state-space model for the state vector $\bar{x}=\left[\begin{array}{ll}i_{L} & v_{c}\end{array}\right]^{T}\left(i_{L}\right.$ the inductor current and $v_{c}$ the capacitor voltage) is described by :

$$
\dot{\bar{x}}=\bar{A} \bar{x}+\bar{B} \bar{u}
$$

with

$$
\bar{A}=\left[\begin{array}{cc}
0 & \frac{1}{L} \\
\frac{1}{C_{c}} & \frac{-1}{R C_{c}}
\end{array}\right], \bar{B}=\left[\begin{array}{c}
\frac{1}{L} \\
0
\end{array}\right], \text { and } \bar{u} \in \overline{\mathscr{V}}=\{0, E\}
$$

Here we consider the numerical values $L=2 \mathrm{mH}, C_{c}=$ $470 \mu \mathrm{F}, E=15 \mathrm{~V}$, and $R=10 \Omega$. One can note that the eigenvalues of the open loop system are purely imaginary $\left( \pm 10^{3} \times\right.$ $1.03 i)$. Here we want to stabilize the system to the equilibrium point $\bar{x}_{e q}=-\bar{A}^{-1} \bar{B} \beta_{e q}$ which correspond to $i_{L}=$ $0.16 \mu \mathrm{A}$ and $v_{c}=7.5 \mathrm{~V}$. Using the transformation from [24] and the change of coordinates $x=\bar{x}-\bar{x}_{e q}$, system (159) becomes

$$
\begin{aligned}
& \dot{x}=A x+B u, \\
& y=C x,
\end{aligned}
$$

with $A=\bar{A}, u \in=\left\{-\frac{1}{2}, \frac{1}{2}\right\}, C=\left[\begin{array}{ll}0 & 1\end{array}\right]$, and $B=\left[\begin{array}{c}\frac{E}{L} \\ 0\end{array}\right]$. We can remark that system (160) satisfies Assumptions A-1, A2 and A-3. Therefore, we can design an observer based relay feedback controller. Considering a decay rate $\alpha=2.55$, LMIs (95)-(96) are feasible with $Q_{1}=\left[\begin{array}{cc}1.33 & -7.79 \\ -7.79 & 55.81\end{array}\right]$,
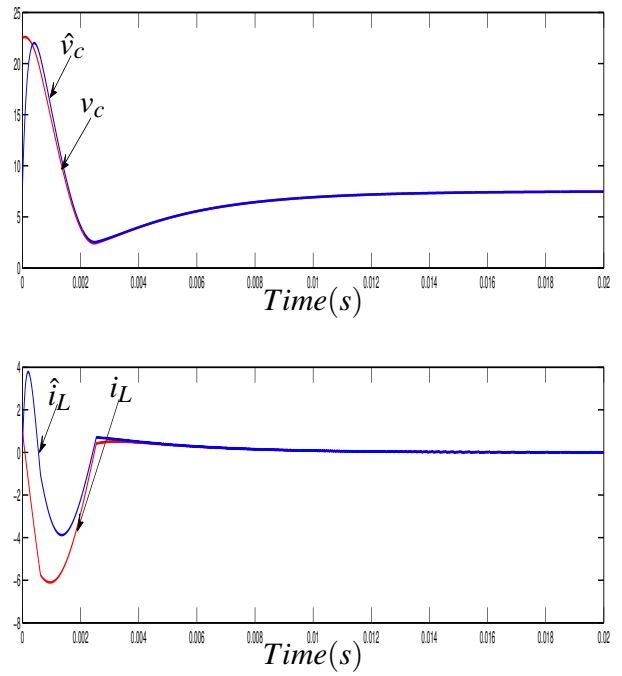

Fig. 5. Evolution of the state variables and their estimates

$P_{2}=\left[\begin{array}{cc}0.005 & -0.002 \\ -0.002 & 0.0015\end{array}\right], \theta_{1}=20.1, \theta_{2}=4.6 \times 10^{6}, \Psi=$
$10^{3} \times\left[\begin{array}{c}2.05 \\ 0.3\end{array}\right], L=10^{3} \times\left[\begin{array}{c}-3.378 \\ -7\end{array}\right]$, and $H=\left[\begin{array}{ll}-0.6 & 0.98\end{array}\right]$. Considering the obtained matrices $H$ and $L$ and $c=\frac{1}{2}$, we can design a nonlinear switching law depending in the estimated states (102). We obtain : $\tilde{P}_{1}=\left[\begin{array}{cc}1.47 & 0.21 \\ 0.21 & 0.037\end{array}\right], \tilde{P}_{2}=10^{8} \times$ $\left[\begin{array}{cc}5.41 & -2.07 \\ -2.07 & 5.27\end{array}\right], \tilde{\Omega}=0.35, \tilde{\Upsilon}=10^{2} \times\left[\begin{array}{ll}-0.72 & 1.075\end{array}\right], \tilde{M}=$ $1.11 \times 10^{2}, \tilde{\tau}=1.24$ with an estimation of the domain of attraction (103) given by $\eta^{-1}=9.94 \times 10^{3}$. Simulations are performed for the initial conditions $\hat{x}=\left[\begin{array}{ll}0 & 0\end{array}\right]^{T}$ and $x=\left[\begin{array}{ll}1 & 15\end{array}\right]^{T}$. The results are reported in Figure 5, which shows that the estimated state converges to the real state and they both converge to the equilibrium point.

\section{Conclusion}

This article presents a new approach for the design of relay feedback controllers. In the case of linear systems, nonquadratic Lyapunov functions are used to develop a method allowing the computation of nonlinear switching surfaces and the enlargement of the domain of attraction. LMI criteria are given in order to design the controller and provide an estimation of a non-ellipsoidal domain of attraction. The problem of observer-based relay feedback controllers design is also considered to ensure local asymptotic stability of the origin of LTI systems. A Luenberger observer is used to design both linear and nonlinear switching surfaces dependent 
on the estimated state. LMI conditions are proposed in order to allow a numerical implementation of the results. The proposed approaches are also extended to the case of stabilization of nonlinear input-affine systems by a relay feedback controller.

\section{References}

[1] B. Aguirre, C. Ibarra, and R. Suárez. Sufficient algebraic conditions for stability of cones of polynomials. Systems \& Control Letters, 46(4):255-263, 2002.

[2] B. Aguirre, J. Solis-Daun, and R. Suárez. Stabilization of linear systems: a polynomial approach. Advances in Dynamics, Instrumentation and Control, 2:21-28, 2007.

[3] A. A. Andronov and W. Fishwick. Theory of oscillators, volume 4. Courier Corporation, 1966.

[4] J. P. Aubin and A. Cellina. Differential inclusions: set-valued maps and viability theory. Springer-Verlag New York, Inc., 1984.

[5] A. Bacciotti and L. Rosier. Liapunov functions and stability in control theory. Springer Science \& Business Media, 2006.

[6] S. Bacha, I. Munteanu, and A. Iuliana Bratcu. Power electronic converters modeling and control. Advanced Textbooks in Control and Signal Processing, 454, 2014.

[7] G. Bartolini, E. Punta, and T. Zolezzi. Simplex sliding mode control of multi-input systems with chattering reduction and mono-directional actuators. Automatica, 47(11):2433-2437, 2011.

[8] F. Blanchini. Survey paper: Set invariance in control. Automatica, 35(11):1747-1767, 1999.

[9] I. Boiko. Discontinuous control systems: frequencydomain analysis and design. Springer Science \& Business Media, 2008.

[10] S. P. Boyd, L. El Ghaoui, E. Feron, and V. Balakrishnan. Linear matrix inequalities in system and control theory, volume 15. SIAM, 1994.

[11] M. S. Branicky. Multiple Lyapunov functions and other analysis tools for switched and hybrid systems. IEEE Transactions on Automatic Control, 43(4):475482, 1998.

[12] J. Cortes. Discontinuous dynamical systems. IEEE Control Systems, 28(3):36-73, 2008.

[13] G. S. Deaecto, J. C. Geromel, F.S. Garcia, and J.A. Pomilio. Switched affine systems control design with application to DC-DC converters. IET Control Theory \& Applications, 4(7):1201-1210, 2010.

[14] M. Defoort, M. Djemai, T. Floquet, and W. Perruquetti. Robust finite time observer design for multicellular converters. International Journal of Systems Science, 42(11):1859-1868, 2011.

[15] R. Delpoux, L. Hetel, and A. Kruszewski. Parameterdependent relay control: Application to PMSM. IEEE Transactions on Control Systems Technology, 23(4):1628-1637, 2015.

[16] C. Edwards and S. Spurgeon. Sliding mode control: theory and applications. CRC Press, 1998.
[17] A. F. Filippov. Differential equations with discontinuous righthand sides: control systems, volume 18 . Springer Science \& Business Media, 1988.

[18] I. Flügge-Lotz. Discontinuous automatic control. Princeton NJ, 1953.

[19] I. Flügge-Lotz. Discontinuous and optimal control. McGraw-Hill, 1968.

[20] R. Goebel, R. G. Sanfelice, and A. R. Teel. Hybrid Dynamical Systems: modeling, stability, and robustness. Princeton University Press, 2012.

[21] J. M. Gonçalves, A. Megretski, M. Dahleh, et al. Global stability of relay feedback systems. IEEE Transactions on Automatic Control, 46(4):550-562, 2001.

[22] W.M. Haddad and T. Sadikhov. Dissipative differential inclusions, set-valued energy storage and supply rate maps, and stability of discontinuous feedback systems. Nonlinear Analysis: Hybrid Systems, 8:83-108, 2013.

[23] C.C. Hang, K.J. Astrom, and Q.G. Wang. Relay feedback auto-tuning of process controllers: a tutorial review. Journal of Process Control, 12(1):143-162, 2002.

[24] L. Hetel and E. Bernuau. Local stabilization of switched affine systems. IEEE Transactions on Automatic Control, 60(4):1158-1163, 2015.

[25] L. Hetel, E. Fridman, and T. Floquet. Sampled-data control of LTI systems with relay: a convex optimization approach. In 9th IFAC Symposium on Nonlinear Control Systems, 2013.

[26] L. Hetel, E. Fridman, and T. Floquet. Variable structure control with generalized relays: A simple convex optimization approach. IEEE Transactions on Automatic Control, 60(2):497-502, 2015.

[27] H. Hindi and S. Boyd. Analysis of linear systems with saturation using convex optimization. In 37th IEEE Conference on Decision and Control, volume 1, pages 903-908, 1998.

[28] K. H. Johansson, A. E. Barabanov, and K. J. Åström. Limit cycles with chattering in relay feedback systems. IEEE Transactions on Automatic Control, 47(9):14141423, 2002.

[29] K. H. Johansson, A. Rantzer, et al. Fast switches in relay feedback systems. Automatica, 35(4):539-552, 1999.

[30] Z. Kader, C. Fiter, L. Hetel, and L. Belkoura. Observerbased relay feedback controller design for LTI systems. In European Control Conference, pages 1667-1672, 2016.

[31] Z. Kader, C. Fiter, L. Hetel, and L. Belkoura. Stabilization of switched affine systems with disturbed statedependent switching. International Journal of Robust and Nonlinear Control, 28(2):582-595, 2018.

[32] H. K. Khalil. Nonlinear systems, volume 3. Prentice hall New Jersey, 1996.

[33] D. Liberzon. Switching in systems and control. Springer Science \& Business Media, 2003.

[34] T. Liu and F. Gao. Industrial process identification and control design: step-test and relay-experiment-based methods. Springer Science \& Business Media, 2011. 
[35] J.-A. Lopez-Renteria, B. Aguirre-Hernandez, and F. Verduzco. The boundary crossing theorem and the maximal stability interval. Mathematical Problems in Engineering, 2011.

[36] D. G. Luenberger. Observers for multivariable systems. IEEE Transactions on Automatic Control, 11(2):190_ 197, 1966.

[37] D.G. Luenberger. Observing the state of a linear system. IEEE Transactions on Military Electronics, 8(2):74-80, April 1964.

[38] B. Paden and S. Sastry. A calculus for computing Filippov's differential inclusion with application to the variable structure control of robot manipulators. IEEE transactions on circuits and systems, 34(1):7382, 1987.

[39] S. R. Parker and S. F. Hess. Limit-cycle oscillations in digital filters. IEEE Transactions on Circuit Theory, 18(6):687-697, 1971.

[40] A. Polyakov. Practical stabilization via relay delayed control. In 47th IEEE Conference on Decision and Control, pages 5306-5311, 2008.

[41] A.E. Polyakov. On practical stabilization of systems with delayed relay control. Automation and Remote Control, 71(11):2331-2344, 2010.

[42] A. Poznyak, A. Polyakov, and V. Azhmyakov. Attractive Ellipsoids in Robust Control. Springer, 2014.

[43] B. Ross Barmish and A. R. Galimidi. Robustness of Luenberger observers: Linear systems stabilized via non-linear control. Automatica, 22(4):413-423, 1986.

[44] R. Schreier, G. C. Temes, and S. R. Norsworthy. DeltaSigma Data Converters: Theory, Design, and Simulation. IEEE, 1997.

[45] S. Tarbouriech, G. Garcia, J. M. G. da Silva Jr, and I. Queinnec. Stability and stabilization of linear systems with saturating actuators. Springer Science \& Business Media, 2011.

[46] S. Tarbouriech, C. Prieur, and J. M. G. da Silva. Stability analysis and stabilization of systems presenting nested saturations. IEEE Transactions on Automatic Control, 51(8):1364-1371, 2006.

[47] I. Z. Tsypkin. Relay control systems. CUP Archive, 1984.

[48] V. Utkin, J. Guldner, and J. Shi. Sliding mode control in electro-mechanical systems, volume 34. CRC press, 2009.

[49] Q.-G. Wang, Tong H. Lee, and L. Chong. Relay feedback: analysis, identification and control. Springer Science \& Business Media, 2012.

[50] C.-C. Yu. Autotuning of PID controllers: A relay feedback approach. Springer Science \& Business Media, 2006.

\section{A Appendix}

Proof of Lemma 3. Let $y \in \mathbb{R}^{m}$ and $i \in \mathscr{I}_{m}$. Three cases arise:

(1) If $y_{(i)}>c_{(i)}$, then $y_{(i)}+\phi_{(i)}\left(y_{(i)}\right)=c_{(i)}$.
(2) If $-c_{(i)} \leq y_{(i)} \leq c_{(i)}$, then $y_{(i)}+\phi_{(i)}\left(y_{(i)}\right)=y_{(i)}$.

(3) If $y_{(i)}<-c_{(i)}$, then $y_{(i)}+\phi_{(i)}\left(y_{(i)}\right)=-c_{(i)}$.

Therefore, for any $i \in \mathscr{I}_{m},\left|y_{(i)}+\phi_{(i)}\left(y_{(i)}\right)\right| \leq c_{(i)}$, and thus $y+\phi(y) \in \mathscr{P}(c)$. 